Draft Version SEPTEMber 20, 2017

Preprint typeset using $\mathrm{LAT}_{\mathrm{E}} \mathrm{X}$ style emulateapj v. 2/19/04

\title{
RESOLVING THE STELLAR POPULATIONS IN THE CIRCUMNUCLEAR RING OF NGC 7469
}

\author{
T. Díaz-Santos ${ }^{1}$, A. Alonso-Herrero ${ }^{1}$, L. Colina $^{1}$, S. D. Ryder ${ }^{2}$, And J. H. Knapen ${ }^{3}$ \\ Draft version September 20, 2017
}

\begin{abstract}
We investigate the stellar populations in the star forming ring of the luminous infrared galaxy NGC 7469. We use Hubble Space Telescope multi-wavelength (UV through NIR) imaging complemented with new $K$-band ground-based long-slit spectroscopy, and mid-IR and radio maps from the literature. Spectral energy distributions (SEDs) and evolutionary synthesis models have been used to characterize the star formation at different scales from those of individual star clusters (tens of pc) to that of the entire star-forming ring (kpc scale). At the smallest scales two different populations of massive $\left(1-10 \times 10^{6} M_{\odot}\right)$ clusters are identified. About $25 \%$ of the clusters are young $(1-3 \mathrm{Myr})$ and extincted ( $A_{V} \approx 3 \mathrm{mag}$ ), whereas the vast majority are of intermediate age ( $\sim 9$ to $\left.20 \mathrm{Myr}\right)$ and less obscured $\left(A_{V} \approx 1 \mathrm{mag}\right)$. At larger (hundreds of $\mathrm{pc}$ ) scale, an analysis of the integrated SED and spectroscopic data of the ring indicates the presence of two stellar populations. The young $(5-6 \mathrm{Myr})$ and obscured stellar population accounts for the $\operatorname{Br} \gamma$ emission and most of the IR luminosity, and about one-third of the stellar mass of the ring. The much less obscured intermediate-age population has properties similar to those of the majority of the (older) $1.1 \mu \mathrm{m}$-selected star clusters. The distribution of these two populations is clearly different and even spatially anti-correlated. The UV-optical-NIR continuum (including the majority of the clusters) of the ring traces mostly the mildly obscured intermediate-age population, while the MIR and radio peaks mark the location of the youngest and obscured star-forming regions. Moreover, the two brightest MIR and radio peaks are spatially coincident with the ends of the nuclear molecular gas bar. This study emphasizes the need for multi-wavelength, high-angular resolution observations to characterize the star formation in the dust-obscured regions commonly present in LIRGs.

Subject headings: galaxies: individual (NGC 7469) — galaxies: clusters: individual — galaxies: star clusters - galaxies: starburst — galaxies: stellar content — infrared: galaxies
\end{abstract}

\section{INTRODUCTION}

Star formation (SF) in Luminous Infrared Galaxies (LIRGs, $10^{11} \leq L_{\mathrm{IR}[8-1000 \mu \mathrm{m}]} \leq 10^{12} \mathrm{~L}_{\odot}$, see Sanders \& Mirabel 1996, and Lonsdale, Farrah \& Smith 2006 for detailed reviews) is one of the most energetic phenomena in the Universe. In a large fraction of local LIRGs the bulk of their SF is generated within their innermost regions (a few kpc), in ring-like structures, mini-spiral arms, and regions of compact emission (Soifer et al. 2001; AlonsoHerrero et al. 2002, 2006a). These regions of SF contain large amounts of molecular gas $\left(\gtrsim 10^{8} \mathrm{M}_{\odot}\right.$, Scoville et al. 1991 Downes \& Solomon 1998; Gao \& Solomon 2004) and dusty, young $(\lesssim 100 \mathrm{Myr})$, massive $\left(10^{5-7} \mathrm{M}_{\odot}\right)$ super star clusters (SSCs; see, among others, Surace, Sanders, \& Evans 2000; Scoville et al. 2000; Alonso-Herrero et al. 2000, 2002; Wilson et al. 2006). The intense SF is often related to past interactions suffered by the host galaxy with a close companion and/or to the presence of a bar (see Knapen 2004 for a review). Both mechanisms are believed to be efficient in driving large amounts of gas towards the nuclear regions.

NGC 7469 is a SABa galaxy with a Seyfert 1 nucleus, located at a distance of $\sim 65 \mathrm{Mpc}\left(c z \simeq 4917 \mathrm{~km} \mathrm{~s}^{-1}\right.$; Heckman et al. $\left.1986 ; \mathrm{H}_{0}=75 \mathrm{~km} \mathrm{~s}^{-1}\right)$. A companion galaxy, IC 5283, located at $\sim 22 \mathrm{kpc}$ (Burbidge, Bur-

\footnotetext{
${ }^{1}$ Departamento de Astrofísica Molecular e Infrarroja, Instituto de Estructura de la Materia, CSIC, E-28006 Madrid, Spain

2 Anglo-Australian Observatory, P.O. Box 296, Epping, NSW 1710, Australia

${ }^{3}$ Centre for Astrophysics Research, University of Hertfordshire, Hatfield, Herts AL10 9AB, UK
}

bidge \& Prendergast 1963; Dahari 1985), is believed to have interacted with NGC 7469 more than $1.5 \times 10^{8} \mathrm{yr}$ ago (Genzel et al. 1995, hereafter G95). NGC 7469 harbors a circumnuclear star-forming ring with an approximate diameter of $5^{\prime \prime}(\sim 1.6 \mathrm{kpc})$ which accounts for approximately two-thirds of the bolometric luminosity of the galaxy. This circumnuclear emission was first detected in the radio and in the near-infrared (NIR) (Ulvestad, Wilson, \& Sramek 1981, and Cutri et al. 1984, respectively). The ring was later observed in the optical (Wilson et al. 1986; Mauder et al. 1994; Malkan, Gorjian, \& Tam 1998), NIR (G95), mid-infrared (MIR, Miles et al. 1994; Soifer et al. 2003; Gorjian et al. 2004), far-infrared (FIR, Papadopoulos et al. 2000), and again at radio wavelengths (Meixner et al. 1990; Wilson et al. 1991). NGC 7469 has a large-scale (several kpc) stellar bar detected in the NIR (Knapen, Shlosman \& Peletier 2000) that gives the galaxy its 'SAB' classification, but also harbors a nuclear gas bar (with a size similar to the diameter of the ring) that has been reported by various authors (Laine et al. 2002; Davies, Tacconi, \& Genzel 2004). A bright radio supernova (SN2000ft) has been discovered in the circumnuclear ring (Colina et al. 2001; Alberdi et al. 2006), and recently its optical counterpart has been identified (Colina et al. 2007).

G95 presented a combination of NIR broad-band speckle and Fabry-Perot imaging, as well as integral-field spectroscopy of the nuclear regions of NGC 7469 at subarcsecond resolution. They were able to separate the emission from the unresolved Seyfert nucleus from that of the surrounding starburst ring. By comparing multi- 
wavelength diagnostics for the ring as a whole with their starburst model, they concluded that SF in the ring has either: (a) been progressing at a constant rate for the past several $10^{7} \mathrm{yr}$, but with few high-mass stars; or (b) been decaying exponentially since the onset of a burst 15 Myr ago, with a fairly 'normal' initial mass function (IMF). However, they were unable to assess the possible variation of the SF history along the ring.

In this paper we present Hubble Space Telescope (HST) UV through NIR imaging, as well as low resolution $(R \sim$ 600) $K$-band spectroscopy at four different locations along the circumnuclear star-forming ring of NGC 7469. Our goal is to study in detail the SF properties of individual star clusters (spatial scales of tens of parsecs) detected in the ring (see, e.g., Scoville et al. 2000), of ring sections (spatial scales of a few hundred parsecs), as well as of the ring as a whole. This work is organized as follows: in $\S 2$ we present imaging and spectroscopic data and their reduction procedures and analysis; in $\S 3$ we describe the fitting method employed to obtain the parameters of the stellar populations; in $\S$ 4 we present the results for the smallest scales (individual clusters) whereas results for the ring sections and the whole ring are given in $\S$, finally, in $\S 6$ we discuss the results and in $\S[$ briefly summarize the main conclusions.

\section{OBSERVATIONS AND DATA ANALYSIS}

\subsection{HST Archival Imaging}

We have retrieved HST archival images of the central region of NGC 7469 taken through eight filters using three different instruments: WFPC2, NICMOS, and ACS. The images cover a broad wavelength range going from the UV $(0.218 \mu \mathrm{m})$ to the NIR $(2.22 \mu \mathrm{m})$. Detailed information about the HST observations is given in Table 1. The UV and optical images were calibrated employing the On The Fly Recalibration (OTFR) system. We reduced and calibrated the NICMOS images as described by Alonso-Herrero et al. (2000). We subtracted theoretical PSFs generated with the TinyTim software ${ }^{4}$ (Krist et al. 1998) for each filter in an attempt to minimize the Seyfert nucleus contribution when analyzing the properties of the ring of SF. The HST images of the central $\simeq 1.6 \mathrm{kpc}$ of NGC 7469 are presented in Fig. 1.

\subsubsection{Star Cluster selection}

Star clusters were selected at $1.1 \mu \mathrm{m}$ (NICMOS F110W filter) because of two main reasons: (a) the NIR spectral region is less affected by dust extinction than UV and optical wavelengths: $A_{U V}(0.22 \mu \mathrm{m}) \sim 3 \times A_{V} \sim$ $20 \times A_{1.1 \mu m}$ and $A_{B(0.33 \mu \mathrm{m})} \sim 2 \times A_{V} \sim 13 \times$ $A_{1.1 \mu \mathrm{m}}$ (Calzetti 1997, CAL hereafter); (b) the F110W filter has the best spatial resolution among the NICMOS filters (see Table 1).

The positions of the star clusters in all the HST images were determined using a procedure similar to the growing region method employed by the HiIPHOT software (Thilker, Braun, \& Walterbos 2000). This search method is also similar to that used by SEXTRACTOR (Bertin \& Arnouts 1996). A $2 \sigma$ threshold above a background selected in four regions in the outskirts of the ring was imposed for cluster identification. However,

\footnotetext{
${ }^{4}$ http://www.stsci.edu/software/tinytim
}

this background was in some cases not representative of the strong, complex and variable "background" emission surrounding the clusters (that is, underlying unresolved emission within the ring acting as a pedestal background). The search method had to be stopped manually when noise peaks began to be identified as positive detections in the images, specially in those with the lowest $\mathrm{S} / \mathrm{N}$ ratio (F547M and $\mathrm{F} 814 \mathrm{~W})$. A total of 30 clusters in the ring of SF was selected at $1.1 \mu \mathrm{m}$. Their locations are given in Table 2 (see also Fig. 2). Out of the $301.1 \mu \mathrm{m}$ star clusters within the ring selected by our method, only five (C1, C3, C5, C6, and C7) were identified by Scoville et al. (2000) using the same NIR dataset.

In order to construct spectral energy distributions (SEDs) of the $1.1 \mu \mathrm{m}$-selected star clusters, we crosscorrelated their positions with those of clusters detected at other wavelengths. Clusters located at distances $\leq$ $1.5 \times \mathrm{FWHM}_{\mathrm{F} 110 \mathrm{~W}}\left(\leq 00^{\prime \prime} 175 \simeq 55 \mathrm{pc}\right)$ were identified as counterparts of the $1.1 \mu \mathrm{m}$ clusters. Because the F330W and F606W images have both good $\mathrm{S} / \mathrm{N}$ and higher spatial resolution than the F110W image, a few $1.1 \mu \mathrm{m}$ clusters were found to have multiple cross-identifications at these wavelengths. Table 3 provides information about cluster identification and cross-correlation. For all the images except those with the highest spatial resolutions (F330W and F606W), most of the clusters identified at a given wavelength are indeed those also selected at $1.1 \mu \mathrm{m}$, even in the UV. The fraction of the F330W and F606W clusters cross-identified at $1.1 \mu \mathrm{m}$ is smaller than in the other filters, but consistent with the larger number of clusters detected in these two filters.

\subsubsection{Cluster photometry}

The cluster intensity was modelled with a 2D Gaussian function whose width was fixed to that measured from theoretical PSFs generated with TinyTim (Krist et al. 1998) for each filter. Additionally, our method fits the emission surrounding the source with a $2 \mathrm{D}$ plane, which is then interpolated to the region where the cluster is located. The region used to fit the background was defined as the area enclosed by a two pixel wide ring with its inner radius containing the $90 \%$ of the TinyTim PSF flux. This is done after rejecting those pixels that exceed the fitted background by $2.75 \sigma$ (i.e., pixels in the background with possible contamination from other clusters). A $\chi^{2}$ minimizing Marquardt method (Bevington \& Robinson 2003) implemented in IDL was used to derive accurate positions and fluxes of the point sources. However, since the HST PSFs are not Gaussians, an aperture correction (filter-dependent) was needed. These corrections were obtained by modelling the individual theoretical PSFs generated with TinyTim in the same way as the star clusters.

In order to estimate the photometric uncertainty of this method, we generated a grid of test images where TinyTim PSFs with random positions and variable intensities were placed. The number density of point sources was approximately 0.01 pixel $^{-2}$. PSFs separated by $<2-3$ pixels were common in the test images, similar to the the cluster density observed in the ring of NGC 7469. We ran simulations varying the background intensity and standard deviation, as well as the PSF intensity with respect to that of the background. We applied our fitting method to measure the PSF fluxes and 

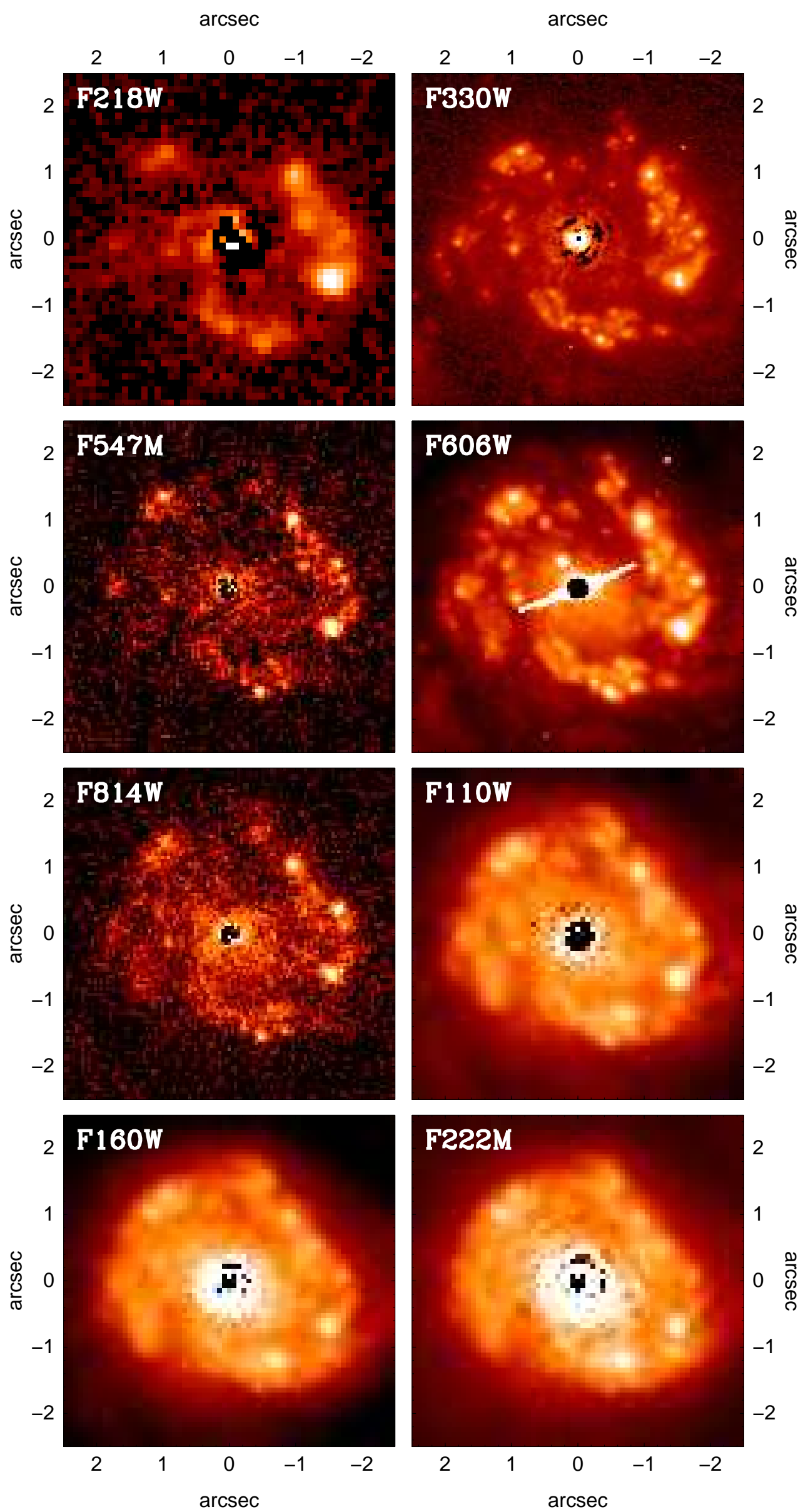
TABLE 1

HST IMAGING DATA

\begin{tabular}{|c|c|c|c|c|c|c|c|c|c|}
\hline Filter & Instrument & Detector & $\begin{array}{c}\text { Plate scale } \\
\left(\operatorname{arcsec}_{\text {pixel }}^{-1}\right)\end{array}$ & $\begin{array}{c}\text { Date } \\
(\mathrm{yy} / \mathrm{mm} / \mathrm{dd})\end{array}$ & $\begin{array}{c}t_{\exp }(a) \\
(s)\end{array}$ & $\begin{array}{c}\text { Orientation } \\
\text { (degrees) }\end{array}$ & $\frac{\text { TT FWHM }^{(\mathrm{b})}}{(\operatorname{arcsec})}$ & $\frac{\text { TT FWHM }^{(\mathrm{b})}}{(\mathrm{pc})}$ & $\mathrm{ID}: \mathrm{PI}^{(\mathrm{c})}$ \\
\hline F218W & WFPC2 & WF3 & 0.1 & $99 / 06 / 28$ & 1200 & 107.54 & 0.176 & 55 & 6358 : Colina \\
\hline F330W & ACS & HRC & 0.026 & $02 / 11 / 20$ & 1140 & -107.75 & 0.051 & 16 & 9379 : Schmitt \\
\hline F547M & WFPC2 & $\mathrm{PC}$ & 0.0455 & $00 / 05 / 13$ & 12 & -61.36 & 0.076 & 24 & 8240 : Wilson \\
\hline F606W & WFPC2 & $\mathrm{PC}$ & 0.0455 & $94 / 06 / 10$ & 500 & -68.35 & 0.073 & 23 & 5479: Malkan \\
\hline F814W & WFPC2 & $\mathrm{PC}$ & 0.0455 & $00 / 05 / 13$ & 7 & -61.36 & 0.085 & 27 & 8240: Wilson \\
\hline F110W & NICMOS & NIC2 & 0.0755 & $97 / 11 / 10$ & 352 & -150.15 & 0.117 & 37 & 7219 : Scoville \\
\hline F160W & NICMOS & NIC2 & 0.0755 & $97 / 11 / 10$ & 352 & -150.15 & 0.154 & 48 & 7219 : Scoville \\
\hline $\mathrm{F} 222 \mathrm{M}$ & NICMOS & NIC2 & 0.0755 & $97 / 11 / 10$ & 480 & -150.15 & 0.202 & 64 & 7219 : Scoville \\
\hline
\end{tabular}

Note. - (a) Total exposure time of the final image; (b) FWHM of TinyTim PSFs; (c) Proposal ID and PI.

TABLE 2

Positions AND FluX Densities OF THE $1.1 \mu$ M-SELECTED STAR Clusters IN THE Ring OF SF

\begin{tabular}{|c|c|c|c|c|c|c|c|c|c|c|}
\hline$\#^{(\mathrm{a})}$ & $X^{(\mathrm{b})}$ & $Y^{(\mathrm{b})}$ & $\mathrm{F} 218 \mathrm{~W}^{(\mathrm{c})}$ & F330W $\mathrm{W}^{(\mathrm{c})}$ & $\mathrm{F} 547 \mathrm{M}^{(\mathrm{c})}$ & $\mathrm{F} 606 \mathrm{~W}^{(\mathrm{c})}$ & $\mathrm{F} 814 \mathrm{~W}^{(\mathrm{c})}$ & $\mathrm{F} 110 \mathrm{~W}^{(\mathrm{c})}$ & $\mathrm{F} 160 \mathrm{~W}^{(\mathrm{c})}$ & $\mathrm{F} 222 \mathrm{M}^{(\mathrm{c})}$ \\
\hline C1 & 54 & -0.67 & $9.88 \times 10^{-16}$ & $1 \# 3.44 \times 10^{-16}$ & $1.03 \times 10^{-16}$ & $7.73 \times 10^{-17}$ & $5.90 \times 10^{-17}$ & $3.58 \times 10^{-17}$ & $2.79 \times 10^{-17}$ & $1.93 \times 10^{-17}$ \\
\hline $\mathrm{C} 2$ & 96 & 0.99 & $.59 \times 10^{-16}$ & $1.69 \times 10^{-16}$ & $1.05 \times 10^{-16}$ & $8.85 \times 10^{-17}$ & $4.95 \times 10^{-17}$ & $2.63 \times 10^{-17}$ & $1.48 \times 10^{-17}$ & $9.85 \times 10^{-18}$ \\
\hline C3 & 93 & 1.34 & $9.41 \times 10^{-17}$ & $7.38 \times 10^{-17}$ & $6.74 \times 10^{-17}$ & $4.94 \times 10^{-17}$ & $2.36 \times 10^{-17}$ & $2.28 \times 10^{-17}$ & $1.79 \times 10^{-17}$ & $1.22 \times 10^{-17}$ \\
\hline $\mathrm{C} 4$ & -0.74 & -1.20 & & $6.87 \times 10^{-18}$ & & $1 \#^{2.96 \times 10^{-17}}$ & & $1.97 \times 10^{-17}$ & $1.93 \times 10^{-17}$ & $1.60 \times 10^{-17}$ \\
\hline C5 & -1.73 & 0.10 & $9.23 \times 10^{-17}$ & $6.42 \times 10^{-17}$ & $4.03 \times 10^{-17}$ & $4.67 \times 10^{-17}$ & $2.27 \times 10^{-17}$ & $1.80 \times 10^{-17}$ & $1.24 \times 10^{-17}$ & $7.93 \times 10^{-18}$ \\
\hline C6 & 1.19 & 1.11 & $4.95 \times 10^{-17}$ & $1 \#^{2.69 \times 10^{-17}}$ & & $2.03 \times 10^{-17}$ & $1.63 \times 10^{-17}$ & $1.68 \times 10^{-17}$ & $1.63 \times 10^{-17}$ & $1.43 \times 10^{-17}$ \\
\hline $\mathrm{C} 7$ & -0.46 & -1.54 & $1.29 \times 10^{-16}$ & $1 \# 1.31 \times 10^{-16}$ & $7.03 \times 10^{-17}$ & $7.59 \times 10^{-17}$ & $3.74 \times 10^{-17}$ & $1.41 \times 10^{-17}$ & & \\
\hline $\mathrm{C} 8$ & .37 & 1.49 & $\ldots$ & $1 \# 8.66 \times 10^{-18}$ & & $1.47 \times 10^{-17}$ & $\ldots$ & $1.34 \times 10^{-17}$ & $1.02 \times 10^{-17}$ & $9.85 \times 10^{-18}$ \\
\hline C9 & .28 & & $\ldots$ & $2 \# 3.23 \times 10^{-17}$ & $1.92 \times 10^{-17}$ & $1 \#^{2.02 \times 10^{-17}}$ & & $1.22 \times 10^{-17}$ & $3 \times 10^{-17}$ & $10^{-18}$ \\
\hline $\mathrm{C} 10$ & .58 & 1.58 & $\ldots$ & $10^{-18}$ & - & $1.31 \times 10^{-17}$ & $\ldots$ & $1.20 \times 10^{-17}$ & $1.23 \times 10^{-17}$ & \\
\hline C11 & 1.27 & -0.68 & $\ldots$ & $2.89 \times 10^{-18}$ & $\ldots$ & & $\ldots$ & $1.09 \times 10^{-17}$ & $1.06 \times 10^{-17}$ & $7.67 \times 10^{-18}$ \\
\hline $\mathrm{C} 12$ & 1.27 & -0.90 & $\ldots$ & $2.89 \times 10^{-18}$ & $\ldots$ & $8.71 \times 10^{-18}$ & $\ldots$ & $1.06 \times 10^{-17}$ & (1) & \\
\hline $\mathrm{C} 13$ & -1.79 & -0.37 & & $1.36 \times 10^{-17}$ & & $1.47 \times 10^{-17}$ & & $1.02 \times 10^{-17}$ & & $4.35 \times 10^{-18}$ \\
\hline C14 & -1.11 & 0.35 & $1.19 \times 10^{-16}$ & $2 \# 6.62 \times 10^{-17}$ & $3.16 \times 10^{-17}$ & $1 \#^{3.70 \times 10^{-17}}$ & $1.65 \times 10^{-17}$ & $1.00 \times 10^{-17}$ & $6.88 \times 10^{-18}$ & $10^{-18}$ \\
\hline $\mathrm{C} 15$ & -0.28 & -1.28 & $3.57 \times 10^{-17}$ & & $2.14 \times 10^{-17}$ & $1 \# 3.64 \times 10^{-17}$ & $1.79 \times 10^{-17}$ & $8.91 \times 10^{-18}$ & $8.28 \times 10^{-18}$ & $7.05 \times 10^{-18}$ \\
\hline $\mathrm{C} 16$ & 1.72 & -0.02 & $3.67 \times 10^{-17}$ & $1 \# 3.11 \times 10^{-17}$ & $\ldots$ & $1.75 \times 10^{-17}$ & $\ldots$ & $8.89 \times 10^{-18}$ & $: 6.81 \times 10^{-18}$ & \\
\hline C17 & 1.04 & 0.37 & & $1.13 \times 10^{-17}$ & $\ldots$ & $1.17 \times 10^{-17}$ & $\ldots$ & $7.82 \times 10^{-18}$ & $6.99 \times 10^{-18}$ & \\
\hline $\mathrm{C} 18$ & 0.17 & -0.92 & $4.22 \times 10^{-17}$ & $2.03 \times 10^{-17}$ & $\ldots$ & $2.29 \times 10^{-17}$ & $\ldots$ & $7.73 \times 10^{-18}$ & $8.36 \times 10^{-18}$ & $6.28 \times 10^{-18}$ \\
\hline C19 & 1.36 & -0.39 & $1.14 \times 10^{-17}$ & $\ldots$ & $\ldots$ & $\ldots$ & $\ldots$ & $7.45 \times 10^{-18}$ & $7.56 \times 10^{-18}$ & $5.93 \times 10^{-18}$ \\
\hline $\mathrm{C} 20$ & -1.49 & -1.05 & $\ldots$ & & $\ldots$ & $\ldots$ & $\ldots$ & $7.35 \times 10^{-18}$ & $8.65 \times 10^{-18}$ & $9.91 \times 10^{-18}$ \\
\hline $\mathrm{C} 21$ & 1.52 & 0.05 & & $3.06 \times 10^{-18}$ & & & & $7.20 \times 10^{-18}$ & $: 6.81 \times 10^{-18}$ & $5.31 \times 10^{-18}$ \\
\hline $\mathrm{C} 22$ & 0.91 & -0.15 & $4.77 \times 10^{-17}$ & $3.15 \times 10^{-17}$ & $3.42 \times 10^{-17}$ & $2.59 \times 10^{-17}$ & $1.77 \times 10^{-17}$ & $6.94 \times 10^{-18}$ & & \\
\hline $\mathrm{C} 23$ & -1.34 & -1.37 & & $1 \# 1.18 \times 10^{-17}$ & otes & $7.69 \times 10^{-18}$ & 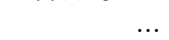 & $6.85 \times 10^{-18}$ & $5.24 \times 10^{-18}$ & \\
\hline $\mathrm{C} 24$ & -1.55 & 0.31 & $4.15 \times 10^{-17}$ & $1 \#: 3.54 \times 10^{-17}$ & $\ldots$ & $1.60 \times 10^{-17}$ & $\cdots$ & $6.18 \times 10^{-18}$ & & \\
\hline $\mathrm{C} 25$ & -1.34 & 0.13 & $\ldots$ & $1 \#: 1.37 \times 10^{-17}$ & $\ldots$ & $7.78 \times 10^{-18}$ & $\cdots$ & $5.97 \times 10^{-18}$ & $4.34 \times 10^{-18}$ & \\
\hline $\mathrm{C} 26$ & 0.90 & 0.24 & $\ldots$ & & ( & $\ldots$ & $\cdots$ & $4.43 \times 10^{-18}$ & & \\
\hline $\mathrm{C} 27$ & 1.29 & 0.51 & $\ldots$ & $2.31 \times 10^{-18}$ & $\ldots$ & & & $4.26 \times 10^{-18}$ & & \\
\hline $\mathrm{C} 28$ & .19 & 1.23 & $\ldots$ & & $\ldots$ & $8.31 \times 10^{-18}$ & & $4.09 \times 10^{-18}$ & $2.87 \times 10^{-18}$ & $3.41 \times 10^{-18}$ \\
\hline C29 & 0.50 & 0.98 & $\ldots$ & $1.48 \times 10^{-17}$ & $\ldots$ & $1.39 \times 10^{-17}$ & & $2.36 \times 10^{-18}$ & 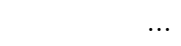 & \\
\hline C30 & -0.98 & -0.28 & $\ldots$ & $\ldots$ & $\ldots$ & 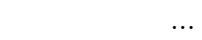 & $\cdots$ & $2.18 \times 10^{-18}$ & & \\
\hline
\end{tabular}

Note. - (a) Cluster label. The clusters are sorted by their F110W flux; (b) Positions of the clusters relative to the nucleus (in arcsec). A positive sign indicates west and north directions; (c) Flux densities (in erg s${ }^{-1} \mathrm{~cm}^{-2} \AA^{-1}$ ) at each filter. The number with a hash symbol next to a flux density indicates the number of additional counterparts that have been detected aside from the first one. The quoted flux is the sum of all the counterparts. A colon next to a flux density indicates that the same cluster is associated with two different $1.1 \mu \mathrm{m}$ star clusters. 


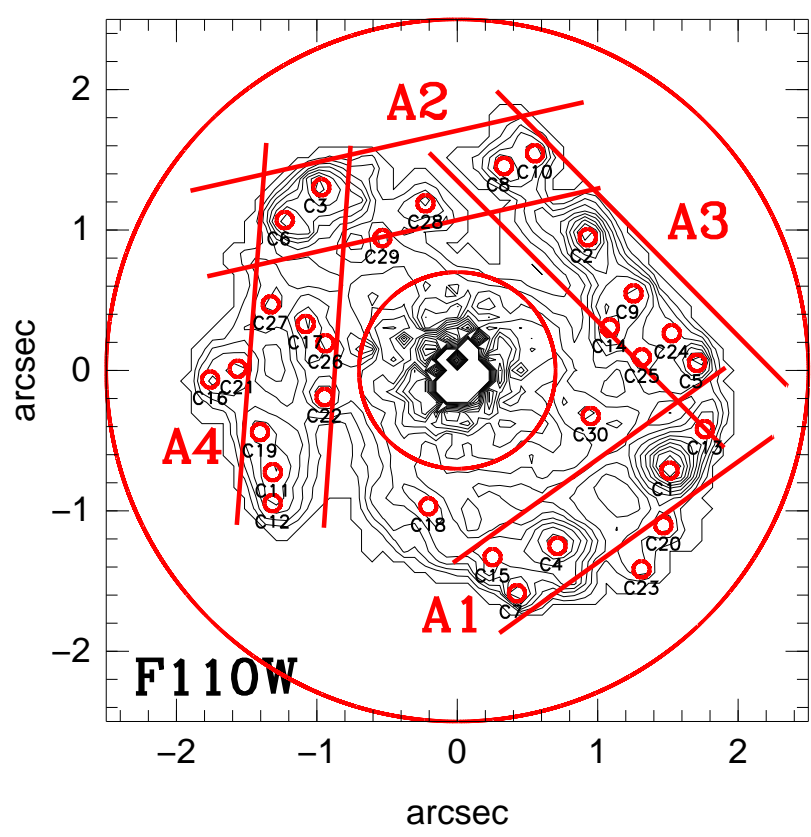

FIG. 2.- The contours, shown in a logarithmic scale, represent the HST/NICMOS F110W emission. The $1.1 \mu$ m-selected star clusters are marked with small open circles with sizes equivalent to the FWHM of the F110W PSF $(\simeq 37 \mathrm{pc}$, see Table 11). The location and P.A. of the UKIRT/CGS4 slits are also marked, wit the $0 . \prime 6$ slit width and extraction lengths (see Table 4) shown to scale. The ring area defined by G95 (enclosed by two open circles of radii 0.7 and $2^{\prime \prime} 5$, see text) is also shown. North is up, and east to the left. [See the electronic edition of the Journal for a color version of this figure.]

TABLE 3

Photometric UnCERTAinties, Number OF Clusters AND CROSS-IDENTIFICATIONS

\begin{tabular}{ccccc}
\hline \hline Filter & $\begin{array}{c}\text { Phot } \\
\text { uncert. (a) }^{(}\end{array}$ & $\begin{array}{c}\text { No. } \\
\text { clusters }^{(b)}\end{array}$ & $\begin{array}{c}\text { No. } \\
\text { cross-ID }^{(c)}\end{array}$ & $\begin{array}{c}\text { Fraction of } \\
1.1 \mu \text { selected }^{(d)}\end{array}$ \\
\hline F218W & $10 \%$ & 16 & 13 & $81 \%$ \\
F330W & $5 \%$ & 56 & $25+11$ & $45+20 \%$ \\
F547M & $15 \%$ & 10 & 9 & $90 \%$ \\
F606W & $5 \%$ & 42 & $23+4$ & $55+10 \%$ \\
F814W & $12 \%$ & 11 & 10 & $91 \%$ \\
F110W & $8 \%$ & 30 & 30 & $100 \%$ \\
F160W & $8 \%$ & 20 & 20 & $100 \%$ \\
F222M & $9 \%$ & 18 & 17 & $94 \%$ \\
\hline
\end{tabular}

Note. - (a) Average estimated uncertainty for the cluster photometry (see text for details); (b) Number of identified clusters; (c) Number of cross-identified $1.1 \mu \mathrm{m}$ clusters in each image plus the number of clusters identified as double or triple counterparts for the F330W and F606W filters; (d) Fraction of cross-identified $1.1 \mu \mathrm{m}$ clusters with respect to the total number of clusters detected in each image.

compare them with the input values. Our point source modelling method was compared with two other classical photometric tools: aperture photometry, and the tasks of DAOPHOT (Stetson 1987) in IRAF ${ }^{5}$. We found that our method is capable of recovering fluxes with an accuracy

5 IRAF is distributed by the National Optical Astronomy Observatories, which are operated by the Association of Universities for Research in Astronomy, Inc., under cooperative agreement with the National Science Foundation.
TABLE 4

UKIRT/CGS4 SLIT PARAMETERS

\begin{tabular}{|c|c|c|c|c|}
\hline Slit & $\begin{array}{c}\text { P.A. } \\
\text { (degree) }\end{array}$ & $\begin{array}{c}t_{\exp }(\mathrm{a}) \\
(\mathrm{s})\end{array}$ & $\begin{array}{l}\text { Aperture }{ }^{(b)} \\
(\operatorname{arcsec})\end{array}$ & $\begin{array}{c}\text { Aperture } \\
(\mathrm{pc})\end{array}$ \\
\hline A1 & 123.9 & 5280 & $0 . \prime 61 \times 2 . .40$ & $192 \times 756$ \\
\hline A2 & 107.7 & 5520 & $0 . \prime 61 \times 2.2^{\prime \prime} 93$ & $192 \times 923$ \\
\hline A3 & 42.1 & 6240 & $0 . \prime 61 \times 33^{\prime \prime} 00$ & $192 \times 945$ \\
\hline $\mathrm{A} 4$ & 174.7 & 5280 & $0 . \prime 61 \times 2.2^{\prime \prime} 69$ & $192 \times 848$ \\
\hline Nucleus & $\ldots$ & 960 & $00^{\prime \prime} 61 \times 11^{\prime \prime} 00$ & $192 \times 315$ \\
\hline
\end{tabular}

Note. - (a) Exposure time; (b) Dimensions of extraction apertures.

typically as high as that of DAOPHOT and in some cases twice as high (depending on the filter and the parameters of the simulation), and $\sim 2-4$ times higher than the classical aperture photometry method. This is probably due to the detailed background modelling performed by our method.

These simulations were also used to estimate the uncertainties (see Table 3) of the cluster photometry in each image by selecting the simulation that best matched the background parameters (standard deviation and relative intensity of the clusters with respect to the background) of the ring. The photometry for the $1.1 \mu \mathrm{m}$-selected star clusters is given in Table 2 In the case of multiple crossidentifications, the sum of the flux densities of all crossidentified clusters at a given wavelength was used as the representative of the cluster selected at $1.1 \mu \mathrm{m}$.

\subsection{Long-slit NIR spectroscopy}

Long-slit spectroscopy of the central region of NGC 7469 was obtained with the common-user NIR spectrograph CGS4 (Mountain et al. 1990) on UKIRT during 1999 October 5 and 6. We employed the 0 ". 61 pixel $^{-1}$ plate scale, a 1 pixel wide slit, and the 40 line $\mathrm{mm}^{-1}$ grating in first order, which delivers complete spectral coverage from $1.85-2.45 \mu \mathrm{m}$ at a resolving power of 600 . Conditions were photometric with $0 . ! 6$ seeing throughout the first night, but increasing amounts of cirrus curtailed observations on the second night. A total of 4 slit position angles and nuclear offsets were selected (see Fig. 2). Details of the observing run are given in Table 4. After setting the slit to the desired position angle, a manual search for the $K$-band nuclear peak was conducted, following which accurate offsets were applied by use of the UKIRT crosshead to place the slit right at the desired locations. In addition, a single slit position passing right through the nucleus was observed.

The data reduction was accomplished using a combination of tasks within the Starlink CGS4DR and NOAO IRAF packages, as described in Ryder et al. (2001). The relatively compact nature of the infrared-emitting central region of NGC 7469 allowed sky subtraction to be performed by sliding the region of interest a total of $38^{\prime \prime} .5$ arcsec along the slit in between 'object' and 'sky' exposures. The difference of each object-sky pair on a given slit position was co-added into groups. The extraction apertures were defined independently for each grouped image, in consultation with Fig. 2, Owing to the still somewhat coarse spatial resolution of CGS4 when compared with the typical size and separation of the clusters in the star- 
forming ring, it proved impossible to reliably extract the emission from individual clusters. Instead, the emission from groups of clusters has been summed, representing the (luminosity-weighted) mean emission from an ensemble of clusters. This nevertheless allows us to compare the emission, and thus the SF history, from four distinct regions along the circumnuclear ring.

\subsubsection{Photometric calibration}

Since the weather conditions were not photometric during the second night, we made use of the available $H S T /$ NICMOS F222M $(\sim K)$ image to perform the photometric calibration of the UKIRT spectra. A TinyTim PSF scaled to best match the bright nuclear emission was subtracted in order to minimize its contamination to the circumnuclear region. We simulated the sizes and orientations of the UKIRT extraction apertures (Table 4) on the F222M image (smoothed to the spatial resolution of the spectra) and measured the flux densities within the four slits. In a similar fashion we measured the fluxes of the spectroscopic apertures on the rest of the HST images in order to obtain the SED of each ring section. The uncertainties of flux densities were estimated by shifting the positions of the simulated apertures \pm 1 pixel in the $x$ and $y$ directions over the HST images, and also by taking into account flux variations within the simulated apertures when we manually chose various intensities to scale the TinyTim PSF with the nuclear peak. Flux densities of the spectroscopic apertures, together with their uncertainties, are given in Table 5. We also obtained integrated photometry through all the HST filters (see Table 2) of the circumnuclear ring region of NGC 7469 for the area enclosed by two circular apertures of radii 0.7 and 2. . 5 , following the analysis of the ring done by G95.

\subsubsection{Spectroscopic analysis}

Fig. 3 shows the spectra of the combined data for each of the four ring sections (apertures), together with the nuclear spectrum. The main spectral features present in all spectra are marked at the top. Line fluxes at $\lambda \leq 2 \mu \mathrm{m}$ have larger intrinsic uncertainties due to attempts to correct for atmospheric transmission, which decreases about $20-40 \%$ from $1.8-1.96 \mu \mathrm{m}$ (these lines are marked with an asterisk in Table 6). Although no systematic under/over-correction of the continuum seems to have been introduced, these values should be taken with caution.

In order to define a consistent continuum right out into the region of extensive $\mathrm{CO}$ absorption beyond $2.3 \mu \mathrm{m}$, we fitted a power-law of the form $F_{\lambda} \propto \lambda^{\beta}$ to featureless sections of the spectrum near 2.09 and $2.25 \mu \mathrm{m}$ (rest wavelength). After normalizing each spectrum by this fit, the equivalent widths (EWs) and fluxes of the most prominent emission features were measured, together with the spectroscopic $\mathrm{CO}_{\text {sp }}$ index, as defined by Doyon, Joseph \& Wright (1994), and the photometric CO index of Frogel et al. (1978): $\mathrm{CO}_{\mathrm{ph}}=\frac{\mathrm{CO}_{\mathrm{sp}}-0.02}{1.46}$. Line fluxes and EWs, as well as the CO indices are presented in Table 6.

The four spectra (see Fig. 3) taken at different positions along the circumnuclear ring are all rather similar both in terms of line fluxes and their EWs. The nuclear spectrum differs from that of the circumnuclear ring pri-

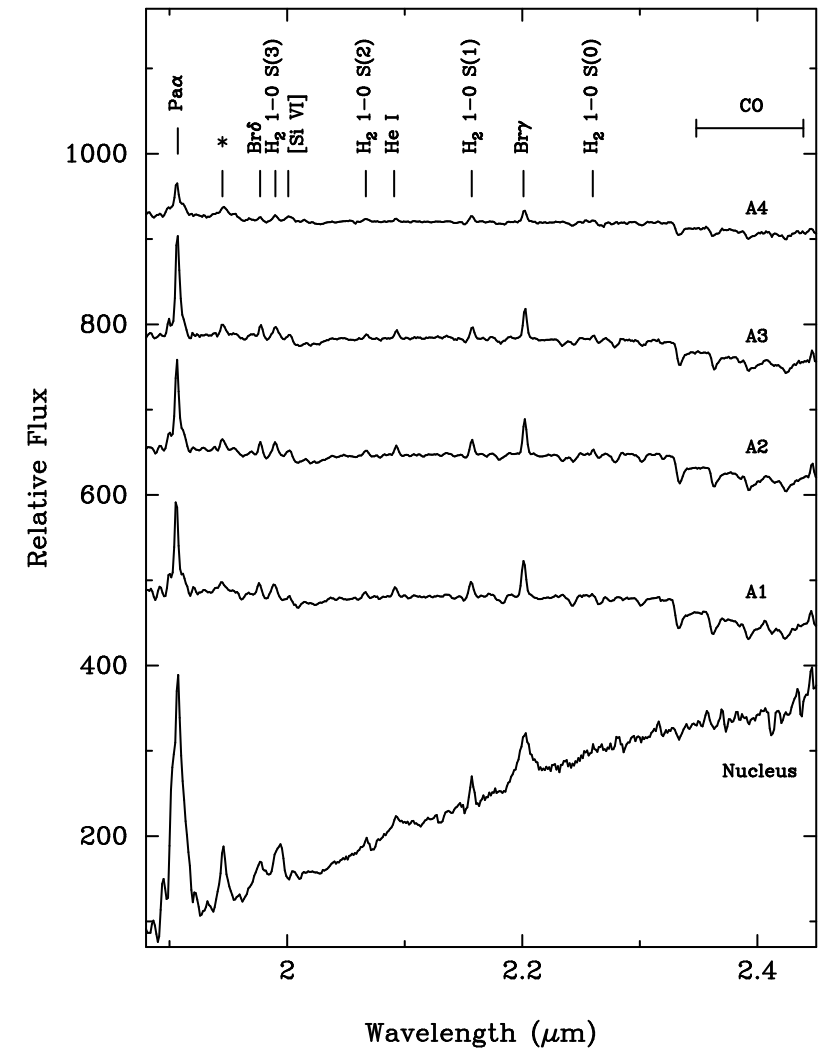

FIG. 3.- Spectra of the four UKIRT slit positions along the circumnuclear ring of NGC 7469, as well as through the Seyfert nucleus. The most prominent emission features are labelled, as well as the CO band. The peak at $1.945 \mu \mathrm{m}$ in each spectrum (marked with an asterisk) is spurious, and arises from $\operatorname{Br} \delta$ absorption intrinsic to the standard star BS 8738 .

marily in two respects: first, the extremely red continuum slope; and second, the broad hydrogen recombination lines. We can use these distinguishing characteristics to place an upper limit on the extent of contamination from the bright nuclear source on the circumnuclear spectra. A contribution of $2 \%$ or more to the integrated $K$-band flux from the nuclear light would be easily noticeable and would have significantly steepened the flat continua of the ring spectra. The absence of even such a small contribution means that the 0".6 slit and UKIRT's active optics facility have done an excellent job at spatially resolving the circumnuclear starburst ring from the Seyfert nucleus, despite the latter being only 1".5 from, but $\sim 3$ mag brighter than, the region sampled by each slit position.

\section{EVOLUTIONARY SYNTHESIS MODELS}

\subsection{Modelling parameters}

We used Starburst99 (SB99 hereafter; Leitherer et al. 1999; Vázquez \& Leitherer 2005; with nebular component included) to model the SF properties of the $1.1 \mu \mathrm{m}$ selected star clusters, the ring sections, and the ring as a whole. The main input parameters are: type of SF, initial mass function (IMF), stellar evolutionary tracks, and metallicity, which was set to solar (a reasonable assumption for nuclear rings, Mazzuca et al. 2006; Allard et al. 2007). As the SF processes taking place in the 
TABLE 5

OBserved Flux Densities of THE SPECTROSCOPIC APERTURES AND THE WhOLE RING

\begin{tabular}{|c|c|c|c|c|c|c|c|c|}
\hline Region & $\mathrm{F} 218 \mathrm{~W}^{(\mathrm{a})}$ & $\mathrm{F}_{330 \mathrm{~W}^{(\mathrm{a})}}$ & $\mathrm{F} 547 \mathrm{M}^{(\mathrm{a})}$ & $\mathrm{F} 606 \mathrm{~W}^{(\mathrm{a})}$ & $\mathrm{F} 814 \mathrm{~W}^{(\mathrm{a})}$ & $\mathrm{F} 110 \mathrm{~W}^{(\mathrm{a})}$ & $\mathrm{F} 160 \mathrm{~W}^{(\mathrm{a})}$ & $\mathrm{F} 222 \mathrm{M}^{(\mathrm{a})}$ \\
\hline A1 & $16.5 \pm 1.0$ & $13.7 \pm 0.5$ & $10.5 \pm 0.2$ & $11.7 \pm 0.2$ & $8.6 \pm 0.3$ & $6.9 \pm 0.3$ & $5.5 \pm 0.2$ & $3.3 \pm 0.1$ \\
\hline $\mathrm{A} 2$ & $3.4 \pm 0.3$ & $4.9 \pm 0.3$ & $6.2 \pm 0.2$ & $7.5 \pm 0.2$ & $6.4 \pm 0.2$ & $6.2 \pm 0.2$ & $5.4 \pm 0.2$ & $3.3 \pm 0.1$ \\
\hline A3 & $11.6 \pm 0.8$ & $11.8 \pm 0.5$ & $10.8 \pm 0.3$ & $11.6 \pm 0.3$ & $9.61 \pm 0.2$ & $7.1 \pm 0.4$ & $5.8 \pm 0.2$ & $3.4 \pm 0.1$ \\
\hline $\mathrm{A} 4$ & $4.5 \pm 0.4$ & $5.8 \pm 0.5$ & $6.8 \pm 0.3$ & $8.9 \pm 0.4$ & $7.4 \pm 0.2$ & $7.1 \pm 0.4$ & $6.1 \pm 0.2$ & $3.6 \pm 0.1$ \\
\hline Ring & $60.6 \pm 2.4$ & $62.2 \pm 1.2$ & $67.9 \pm 2.0$ & $73.6 \pm 2.2$ & $63.0 \pm 1.9$ & $54.3 \pm 1.6$ & $46.5 \pm 1.9$ & $27.9 \pm 0.8$ \\
\hline
\end{tabular}

Note. - (a) Flux densities and uncertainties $\left(10^{-16} \mathrm{erg} \mathrm{s}^{-1} \mathrm{~cm}^{-2} \AA^{-1}\right)$ for each $H S T$ filter.

TABLE 6

Observed line fluXes, EWs AND CO IndiCEs of UKIRT SPECTRA.

\begin{tabular}{|c|c|c|c|c|c|c|c|c|c|}
\hline Line $^{(a)}$ & $\lambda_{0}{ }^{(b)}$ & $\begin{array}{c}\text { A1 } \\
\text { Flux }^{(c)}\end{array}$ & $\begin{array}{c}\mathrm{A} 1 \\
\mathrm{EW}^{(\mathrm{d})}\end{array}$ & $\begin{array}{c}\text { A2 } \\
\text { Flux }^{(c)}\end{array}$ & $\begin{array}{c}\mathrm{A} 2 \\
\mathrm{EW}^{(\mathrm{d})}\end{array}$ & $\begin{array}{c}\text { A3 } \\
\text { Flux }^{(c)}\end{array}$ & $\begin{array}{c}\mathrm{A} 3 \\
\mathrm{EW}^{(\mathrm{d})}\end{array}$ & $\begin{array}{c}\mathrm{A} 4 \\
\text { Flux }^{(\mathrm{c})}\end{array}$ & $\begin{array}{c}\mathrm{A} 4 \\
\mathrm{EW}^{(\mathrm{d})}\end{array}$ \\
\hline $\mathrm{Pa} \alpha^{*}$ & 1.8751 & $7.97 \pm 0.41$ & $23.7 \pm 1.6$ & $9.57 \pm 0.35$ & $28.6 \pm 1.4$ & $12.6 \pm 1.0$ & $36.6 \pm 3.0$ & $9.94 \pm 0.90$ & $25.7 \pm 2.8$ \\
\hline $\operatorname{Br} \delta^{*}$ & 1.9451 & $1.34 \pm 0.20$ & $4.10 \pm 0.70$ & $1.29 \pm 0.30$ & $4.00 \pm 1.00$ & $1.73 \pm 0.28$ & $4.90 \pm 0.90$ & $1.56 \pm 0.24$ & $4.30 \pm 0.70$ \\
\hline $\mathrm{H}_{2} 1-0 \mathrm{~S}(3)^{*}$ & 1.9576 & $1.84 \pm 0.16$ & $5.70 \pm 0.50$ & $1.91 \pm 0.24$ & $6.00 \pm 0.80$ & $2.25 \pm 0.23$ & $6.70 \pm 0.70$ & $2.33 \pm 0.26$ & $6.50 \pm 0.70$ \\
\hline$[\mathrm{SiVI}]^{*}$ & 1.9645 & $0.35 \pm 0.05$ & $1.10 \pm 0.15$ & $0.90 \pm 0.13$ & $2.90 \pm 0.55$ & $0.60 \pm 0.08$ & $1.80 \pm 0.25$ & $0.67 \pm 0.14$ & $1.90 \pm 0.40$ \\
\hline $\mathrm{H}_{2} 1-0 \mathrm{~S}(2)$ & 2.0338 & $0.66 \pm 0.07$ & $2.00 \pm 0.20$ & $0.73 \pm 0.14$ & $2.30 \pm 0.50$ & $0.56 \pm 0.07$ & $1.70 \pm 0.20$ & $0.95 \pm 0.15$ & $2.70 \pm 0.40$ \\
\hline HeI & 2.0581 & $0.95 \pm 0.10$ & $2.90 \pm 0.30$ & $0.98 \pm 0.08$ & $3.10 \pm 0.30$ & $1.04 \pm 0.06$ & $3.10 \pm 0.20$ & $0.66 \pm 0.14$ & $1.80 \pm 0.40$ \\
\hline $\mathrm{H}_{2} 1-0 \mathrm{~S}(1)$ & 2.1218 & $1.30 \pm 0.09$ & $3.90 \pm 0.30$ & $1.47 \pm 0.09$ & $4.50 \pm 0.30$ & $1.38 \pm 0.06$ & $4.10 \pm 0.20$ & $1.44 \pm 0.13$ & $4.10 \pm 0.20$ \\
\hline $\operatorname{Br} \gamma$ & 2.1661 & $3.51 \pm 0.13$ & $10.7 \pm 0.40$ & $3.72 \pm 0.12$ & $11.5 \pm 0.40$ & $3.60 \pm 0.11$ & $10.6 \pm 0.40$ & $3.00 \pm 0.11$ & $8.40 \pm 0.35$ \\
\hline $\mathrm{H}_{2} 1-0 \mathrm{~S}(0)$ & 2.2233 & $0.15 \pm 0.05$ & $1.45 \pm 0.15$ & $0.47 \pm 0.12$ & $1.45 \pm 0.40$ & $0.39 \pm 0.07$ & $1.16 \pm 0.20$ & $0.36 \pm 0.06$ & $1.02 \pm 0.20$ \\
\hline $\mathrm{CO}_{\mathrm{sp}}$ & 2.35 & & $0.21 \pm 0.05$ & & $0.23 \pm 0.05$ & 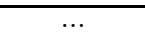 & $0.23 \pm 0.05$ & & $0.20 \pm 0.05$ \\
\hline $\mathrm{CO}_{\mathrm{ph}}$ & 2.35 & $\ldots$ & $0.16 \pm 0.05$ & $\ldots$ & $0.17 \pm 0.05$ & $\ldots$ & $0.17 \pm 0.05$ & $\ldots$ & $0.15 \pm 0.05$ \\
\hline
\end{tabular}

Note. - (a) Emission lines and CO indices; (b) Rest line/band wavelength $(\mu \mathrm{m}) ;(\mathrm{c}) \mathrm{Flux}\left(10^{-15} \mathrm{erg} \mathrm{s}^{-1} \mathrm{~cm}^{-2}\right) \mathrm{within}^{\mathrm{each}}$ aperture; (d) EW in the case of emission lines $(\AA)$; photometric and spectroscopic CO indices in the case of CO bands (see text); * Line fluxes with large uncertainties due to poor atmospheric transmission shortward of $2 \mu \mathrm{m}$ (see text).

nuclear regions of LIRGs and ULIRGs are believed to last only a few tens of Myr (Kennicutt 1998, Allard et al. 2006, 2007), we used instantaneous SF bursts. For the IMF we have used a Salpeter (1955) IMF with lower and upper mass limits of $0.1 \mathrm{M}_{\odot}$ and $100 \mathrm{M}_{\odot}$, respectively. Also, because the star clusters located in the circumnuclear ring of NGC 7469 are expected to be young ( 1 - 50 Myr - since about $80 \%$ of the $1.1 \mu \mathrm{m}$-selected clusters are UV-emitters-, Table [3] see also G95), we have used the tracks from the Geneva group because they better reproduce the SEDs of young, ionizing stellar populations (Vázquez \& Leitherer 2005).

We have constructed a grid of theoretical SEDs with ages ranging from 0.5 to $500 \mathrm{Myr}$. The steps of this grid are 0.5 Myr for the $1-10$ Myr age interval, 1 Myr for 10 - 20 Myr, 5 Myr for 20 - 100 Myr, and 50 Myr for 100 - $500 \mathrm{Myr}$. The model spectra were reddened assuming the extinction law of Calzetti et al. 1997 (CAL) in a simple dust screen configuration which is a good representation of the dust geometry of individual clusters. This dust screen model, however, may not be appropriate for extended regions where more complicated dust geometries are expected (see discussion by G95).

\subsection{Fitting Method}

We have compared observational and theoretical SEDs through a $\chi^{2}$-fitting procedure (Bik et al. 2003):

$$
\chi_{i, j, k}^{2}\left(t_{i}, A_{V j}, m_{*, k}\right)=\sum_{N} \frac{\left(f_{\text {obs }}-f_{\text {model }}\right)^{2}}{\sigma_{\text {obs }}^{2}}
$$

where $N$ are the filters or observables in general (photometric flux densities, flux and/or EW of emission lines, etc.) available for each cluster, ring section, or entire ring, $f_{\text {obs }}$ and $f_{\text {model }}$ are the observed and model quantities respectively, and $\sigma_{\text {obs }}$ are the weights for the fit. In our case, $\sigma_{\text {obs }}$ accounts for the photometric calibration uncertainties, as well as the uncertainties associated with the theoretical models. The $\chi^{2}$ minimization method for fitting SEDs produces more satisfactory results for determining the ages, extinctions, and masses of star clusters detected in galaxies (see, e.g., Maoz et al. 2001; Bastian et al. 2005; de Grijs et al. 2005) than other widely used methods (e.g., color-color diagrams).

When fitting the photometric SEDs (see next sections) we used flux densities instead of magnitudes to avoid the uncertainties associated with transforming fluxes based on the $H S T$ photometric system to the standard Johnson-Cousins ( $U B V I J H K)$ system (see discussion by de Grijs et al. 2005). To compare the observed flux densities with the outputs of the evolutionary models, the model spectra (which include the nebular contribution and the main hydrogen recombination lines) were convolved with the eight HST filter bandpasses that take into account both the filter response and the cameratelescope system throughput. For the model uncertainties we take $5 \%$ for the optical and NIR, and $10 \%$ for the UV (as assumed by de Grijs et al. 2005).

In the case of a single stellar population, there is a total of three parameters to be fitted: age $(t)$, extinction $\left(A_{V}\right)$, and stellar mass $\left(m_{*}\right)$. To be precise, the stellar mass is 
TABLE 7

Results of the $\chi^{2}$-FitTing of the $1.1 \mu \mathrm{m}$-Selected Cluster SEDs.

\begin{tabular}{|c|c|c|c|c|c|}
\hline Cluster & $\begin{array}{l}t^{(\mathrm{a})} \\
(\mathrm{Myr})\end{array}$ & $\begin{array}{l}A_{V}(\mathrm{~b}) \\
(\mathrm{mag})\end{array}$ & $\begin{array}{c}m_{*}(\mathrm{c}) \\
\left(\times 10^{6} \mathrm{M}_{\odot}\right)\end{array}$ & $\chi^{2(\mathrm{~d})}$ & $\nu^{(\mathrm{e})}$ \\
\hline $\mathrm{C} 1_{1 \#}$ & $13.0 \pm_{1}^{1.0}$ & $0.00 \pm_{0}^{0.25}$ & $5.9 \pm_{0.2}^{0.2}$ & 51.74 & 5 \\
\hline $\mathrm{C} 2$ & $20.0 \pm 5: 0$ & $0.50 \pm 0.25$ & $11.3 \pm_{0}^{1.5}$ & 11.80 & 5 \\
\hline C3 & $17.0 \pm_{1.0}^{13.0}$ & $1.25 \pm_{0}^{0.25}$ & $11.5 \pm 1.3$ & 28.78 & 5 \\
\hline $\mathrm{C} 4_{1 \#}$ & $2.0 \pm 0.5$ & $4.50 \pm_{0.25}^{0.25}$ & $16.1 \pm_{3.0}^{0.2}$ & 5.54 & 2 \\
\hline C5 & $17.0 \pm 10^{18.0}$ & $1.25 \pm_{0.50}^{0.25}$ & $9.7 \pm \frac{1.3}{2}$ & 17.08 & 5 \\
\hline $\mathrm{C} 6_{1 \#}$ & $12.0 \pm 1.0$ & $1.00 \pm_{0.25}^{8.50}$ & $3.1 \pm_{1.2}^{0.6}$ & 27.98 & 4 \\
\hline $\mathrm{C} 77_{1 \#}$ & $3.0 \pm \pm_{1}^{0.5}$ & $1.75 \pm_{0.25}^{0.25}$ & $4.8 \pm_{10}^{0: 4}$ & 9.92 & 3 \\
\hline $\mathrm{C} 8_{1 \#}$ & $17.0 \pm_{3.0}^{23.0}$ & $2.75 \pm_{0.75}^{0.25}$ & $\begin{array}{r}11.9 \pm_{7.2}^{3.5} \\
1.5\end{array}$ & 13.76 & 2 \\
\hline $\mathrm{C} 9_{3 \#}$ & $8.5 \pm_{0.5}^{4.5}$ & $0.50 \pm_{0.25}^{0.50}$ & $1.1 \pm_{0 .}^{1} \cdot 9^{2}$ & 27.88 & 3 \\
\hline $\mathrm{C} 10$ & $20.0 \pm 80.0$ & $3.00 \pm 0.25$ & $13.0 \pm \frac{18.2}{18}$ & 2.75 & 1 \\
\hline C11 & $11.0 \pm 19.0$ & $2.50 \pm_{0.25}^{1.00}$ & $2.5 \pm_{0.6}^{11.3}$ & 2.34 & 1 \\
\hline $\mathrm{C} 13$ & $16.0 \pm \pm_{80}^{1.5 .0}$ & $1.75 \pm_{0.75}^{0.25}$ & $4.8 \pm 1.8$ & 0.46 & 1 \\
\hline $\mathrm{C} 14_{3 \#}$ & $17.0 \pm \pm_{16.5}^{23.0}$ & $0.75 \pm_{0.50}^{1: 25}$ & $4.9 \pm 1.3$ & 35.06 & 5 \\
\hline C15 $1 \#$ & $1.0 \pm 0.5$ & $2.25 \pm_{0.25}^{0.50}$ & $3.7 \pm 0.5$ & 25.36 & 4 \\
\hline $\mathrm{C} 16_{1 \#}$ & $17.0 \pm 13.0$ & $1.25 \pm_{0.50}^{0.25}$ & $4.5 \pm_{1}^{0: 4}$ & 6.30 & 2 \\
\hline $\mathrm{C} 17^{1 \pi}$ & $17.0 \pm 9.0$ & $2.00 \pm_{1.00}^{0.25}$ & $5.4 \pm_{4.6}^{0.3}$ & 2.76 & 1 \\
\hline C18 & $8.5 \pm_{0.5}^{4.5}$ & $0.75 \pm_{0}^{0.25}$ & $0.8 \pm_{0}^{1.0}$ & 31.59 & 3 \\
\hline C19 & $12.0 \pm \frac{1.0}{3}$ & $1.50 \pm_{025}^{0.25}$ & $1.6 \pm_{0}^{0: \frac{2}{2}}$ & 11.06 & 1 \\
\hline $\mathrm{C} 21$ & $2.0 \pm_{1}^{18} 8$ & $4.25 \pm_{2.25}^{0.25}$ & $5.2 \pm_{4}^{2.8}$ & 5.35 & 1 \\
\hline $\mathrm{C} 22$ & $200.0 \pm^{50.0}$ & $0.00 \pm 1.25$ & $12.0 \pm_{105}^{0.2}$ & 3.26 & 3 \\
\hline $\mathrm{C} 23_{1 \#}$ & $8.5 \pm_{0.5}^{0.5}$ & $0.75 \pm_{0.25}^{0.25}$ & $0.6 \pm_{0.1}^{0.1}$ & 1.17 & 1 \\
\hline $\mathrm{C} 24_{1 \#}$ & $18.0 \pm_{140}^{42.0}$ & $0.75 \pm_{0.50}^{0.75}$ & $2.6 \pm_{1.8}^{1: 8}$ & 7.06 & 1 \\
\hline $\mathrm{C} 25_{1 \#}$ & $8.5 \pm_{0.5}^{4.5}$ & $0.50 \pm_{0.25}^{0.25}$ & $0.5 \pm_{0.1}^{0.6}$ & 1.88 & 1 \\
\hline $\mathrm{C} 28^{1+}$ & $2.0 \pm \pm_{1.5}^{0.5}$ & $3.50 \pm_{0.25}^{0.25}$ & $2.3 \pm_{0.4}^{0.1}$ & 7.12 & 2 \\
\hline
\end{tabular}

Note. - Results of the fits using SB99 models with an instantaneous SF burst, Geneva tracks, and CAL extinction law; (a) Age; (b) Visual extinction; (c) Stellar mass; (d) Best (lowest) $\chi^{2}$ value (see Equation 1); (e) Degrees of freedom. The number with a hash symbol next to a cluster label indicates the number of total additional counterparts that have been detected among all of the images (i.e., taken into account all wavelengths; see Table 2 for details).

a semi-free parameter because the fitting method simply scales the theoretical SEDs with the mass until the maximum likelihood is reached. However, since the mass affects the solution and accuracy of the fit, it is necessary to include it in the equation that describes the method. The expected (minimum) $\chi^{2}$ value of the best fit should be equal to the number of degrees of freedom $(\nu=N-3)$, but in general our fits provide a higher value of $\chi^{2}$ (see Table 7. and next section). Thus, to estimate the uncertainty of the fitted parameters, we define $\nu^{\prime}=\chi_{\min }^{2}$, where $\chi_{\min }^{2}$ is the minimum value obtained for a given cluster. To determine the range of acceptable solutions we take all those solutions within $\chi_{\min }^{2} \pm \Delta \chi_{\min }^{2}$, with $\Delta \chi_{\min }^{2}=\sqrt{2 \cdot \nu^{\prime}}$. This would be equivalent to taking the $\pm 1 \sigma$ solutions. Although this expression is not formally correct, it provides an estimate of the acceptable solutions when fitting the SEDs.

\section{SMALL-SCALE STAR FORMATION: $1.1 \mu \mathrm{M}$-SELECTED MASSIVE STAR CLUSTERS}

We have fitted the photometric SEDs of the $1.1 \mu \mathrm{m}$ selected star clusters using a single stellar population to derive their ages, extinctions, and masses. Since the number of degrees of freedom is $\nu=N-3$, we have only fitted those $1.1 \mu \mathrm{m}$-selected star clusters cross-identified in another three wavelengths or more; this resulted in a total of 24 fitted clusters (see Table 7). Fig. 4 shows the observational data and the best SED fits of the 10 most luminous clusters sorted by their $1.1 \mu \mathrm{m}$ brightness, as well as four fainter clusters with 3 or more degrees of freedom. We also show in this figure the acceptable range of solutions defined as the area between the SEDs of the youngest and oldest valid models (within the $1 \sigma$ uncertainty of the best $\chi^{2}$ value, as explained in $\S 3.2$ ).

The majority $(75 \%)$ of clusters have SEDs best fitted with ages ranging between 8.5 and $20 \mathrm{Myr}$. The upper limits of acceptable ages for these clusters are less constrained because the model SEDs in the $\sim 10-30 \mathrm{Myr}$ range do not vary as quickly as for younger $(\sim 1-10 \mathrm{Myr})$ stellar populations. We also note that clusters not detected in the UV tend to show large uncertainties in their fitted parameters. Nearly all of the remaining $(\sim 21 \%)$ clusters are fitted with a younger, $t=1-3 \mathrm{Myr}$, stellar population. Only one cluster appears to be relatively old (C22, $200 \mathrm{Myr}$ ), although with a wide range of acceptable ages (see Table 7). The ages of the $1.1 \mu \mathrm{m}$ selected clusters are similar to those of clusters detected in other star-forming circumnuclear rings (Maoz et al. 2001; Alonso-Herrero et al. 2001a), although older clusters like C22 ( $\gtrsim 100 \mathrm{Myr}$ ) have also been detected in ringed galaxies (Buta et al. 2000).

Fig. 5 shows the age distribution of the clusters as a function of their position angle within the ring of SF. As well as in age, the two groups of star clusters also appear to be well differentiated in terms of their extinction (see Table 7). The youngest (2.5 Myr on average) have a mean extinction of $A_{V} \sim 3 \mathrm{mag}$, whereas those of intermediate age (on average $14 \mathrm{Myr}$ ) tend to be less obscured (mean extinction of $A_{V} \sim 1.25 \mathrm{mag}$ ). Some clusters (C1, C2, C3, C6, C8, C9, C14, C15, C18 and C19) present a $2.2 \mu \mathrm{m}$ excess $(\sim 20 \%)$ with respect to their fitted SEDs. Maoz et al. (2001) found a similar behavior in clusters detected in NGC 1512 and NGC 5248, and interpreted it as an additional emission component from dust heated by the star clusters. The spatial distribution of the $1.1 \mu \mathrm{m}$-selected star clusters and their relation to the gas and dust distribution in the ring will be discussed in detail in $\S 6$.

A large fraction of clusters in the ring of NGC 7469 has stellar masses between 0.5 and $10 \times 10^{6} \mathrm{M}_{\odot}$, although about one-third appear to be more massive $(\geq$ $\left.10 \times 10^{6} \mathrm{M}_{\odot}\right)$. These values are also in agreement with stellar masses inferred for SSCs found in other galaxies $\left(10^{4} \mathrm{M}_{\odot} \lesssim \mathrm{M} \lesssim 10^{6} \mathrm{M}_{\odot}\right.$ for M82, de Grijs, Parmentier \& Lamers 2005) and especially in LIRGs (from $\sim 10^{4} \mathrm{M}_{\odot}$ to few $10^{7} \mathrm{M}_{\odot}$; Antennae galaxy, Zhang \& Fall 1999; NGC 3256 and others, Alonso-Herrero et al. 2000, 2001b, 2002; Arp 299, García-Marín et al. 2006; Arp 220, Wilson et al. 2006). Conversely, other studies suggest that not all circumnuclear rings show such massive SSCs (Maoz et al. 1996; Maoz et al. 2001), although these galaxies are not classified as LIRGs. One possibility is that some of the $1.1 \mu \mathrm{m}$-selected clusters identified as point sources in the ring of NGC 7469 are aggregates of less massive clusters, as suggested by the ACS F330W image (see Fig. 1).

\section{STAR FORMATION ON THE KILOPARSEC SCALE: THE CIRCUMNUCLEAR RING}

\subsection{Stellar Populations}

The four UKIRT slits (see Fig. 2) cover regions with sizes of the order of $0.2 \mathrm{kpc} \times 1.4 \mathrm{kpc}$ each, increasing 


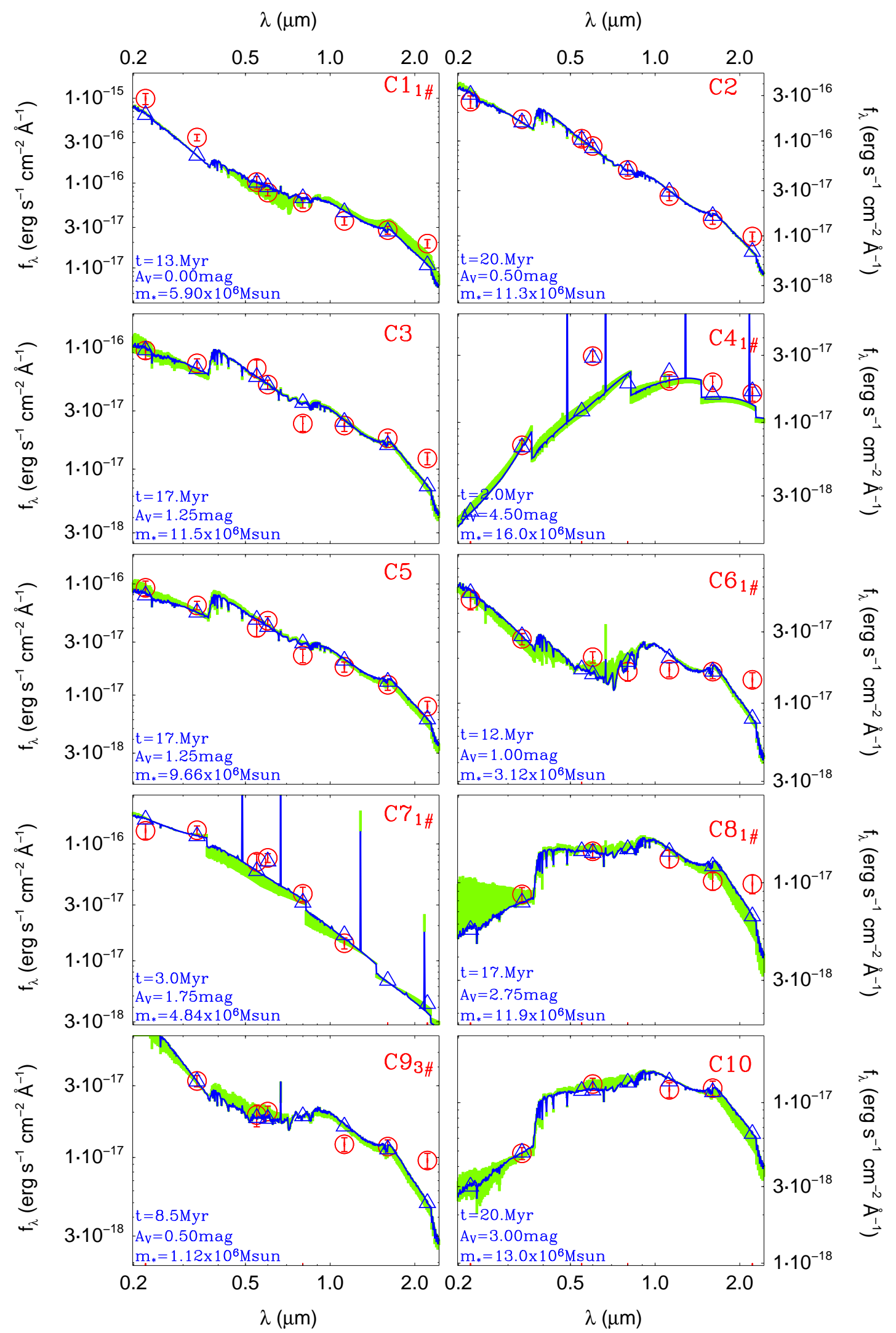

FIG. 4.- Fits to the $1.1 \mu \mathrm{m}$-selected star cluster photometric SEDs. The model SEDs (SB99) use instantaneous star formation, the Geneva tracks, and the CAL extinction law. The ten brightest clusters are shown as well as the remaining four clusters with more than 3 degrees of freedom (see Table 7) are shown as (red) open circles with their $1 \sigma$ uncertainties. The (blue) solid line is the best fit of the observational datapoints, and the open, (blue) triangles represent the photometric points resulting from convolving the best fitted SED with the HST-filter throughputs. The (green) shaded regions represent the SED range of the youngest and oldest acceptable solutions (Table 7). The emission lines seen in the youngest SEDs are hydrogen recombination lines included in the modelling. As in Table 7 the number with a hash symbol next to a cluster label indicates the number of total additional counterparts that have been detected among all of the image. [See the electronic edition of the Journal for a color version of this figure.] 


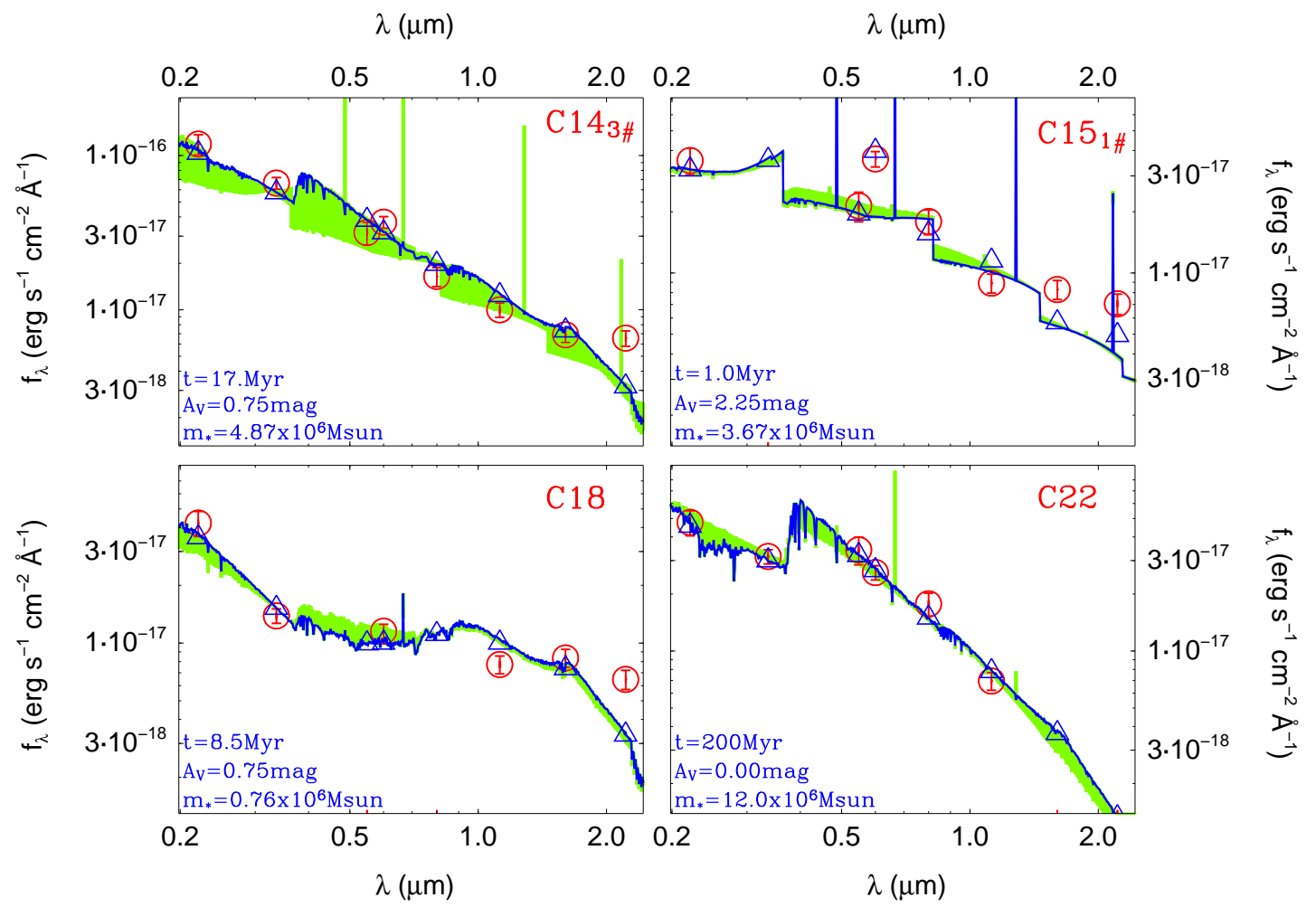

FIG. 4.- Continued. [See the electronic edition of the Journal for a color version of this figure.] 


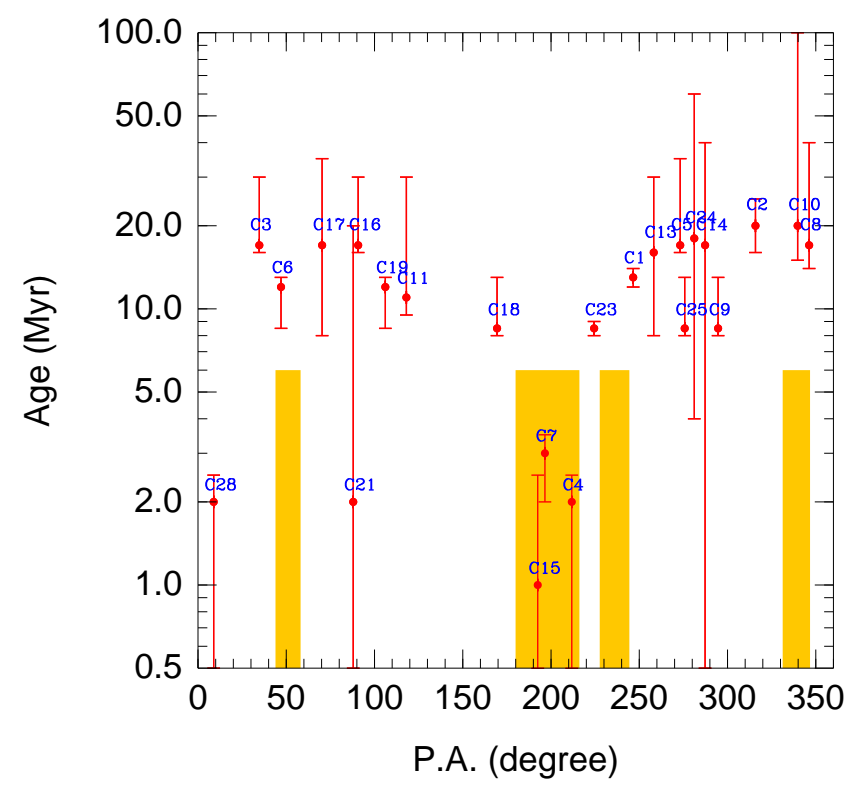

FIG. 5. - Ages of the $1.1 \mu \mathrm{m}$-selected star clusters as a function of their P.A. within the circumnuclear ring. The position angle is measured so that north is 0 degrees and east is 90 degrees. The shaded regions indicate the locations of the MIR/radio (see Fig. 9) peaks tracing the youngest stars $(\lesssim 6-8 \mathrm{Myr})$ in the SF ring. [See the electronic edition of the Journal for a color version of this figure.]

the likelihood to probe stellar populations with different ages. As discussed in $\S 4$, a large fraction of the $1.1 \mu \mathrm{m}-$ selected star clusters have an average age of $14 \mathrm{Myr}$. Nevertheless, the intense $\mathrm{Br} \gamma$ hydrogen recombination line emission measured from the UKIRT spectra has to be produced by a younger ionizing stellar population. Thus, to fit the photometric SEDs and spectroscopic data $(\mathrm{Br} \gamma$ flux and its EW) of the ring sections we used a simple combination of two stellar populations ${ }^{6}$. Because the ionizing and the MIR emitting regions are spatially coincident (Soifer et al. 2003 and G95, and discussion in $\S 6$ ), the young stellar population has to be at least moderately obscured. Thus, we introduced an additional constraint for the extinction of the young stellar population: $A_{V} \geq 3 \mathrm{mag}$, based on the typical extinctions over the $\mathrm{Pa} \alpha$ emitting regions found for local LIRGs (Alonso-Herrero et al. 2006a). From gas and stellar dynamics, G95 inferred a dynamical mass for the ring of $4.5 \times 10^{9} \mathrm{M}_{\odot}$ whereas Davies et al. (2004) estimated a dynamical mass of $6.5 \times 10^{9} \mathrm{M}_{\odot}$ within a radius of 2.5 " (with the nucleus contributing at most $\sim 15 \%$ ). In addition, G95 estimated a total stellar mass of $2.7 \times 10^{9} \mathrm{M}_{\odot}$ inside the ring. Thus, taking into accout the uncertainties in all the derived masses, we imposed that the total stellar mass fitted for the ring sections (and the whole ring, see below) was not greater than $3.5 \times 10^{9} \mathrm{M}_{\odot}$ ( $\sim 50 \%$ of $\left.M_{\mathrm{dyn}}\right)$. All the solutions that did not obey this condition were discarded.

\footnotetext{
${ }^{6}$ We note that a single stellar population cannot simultaneously fit the photometric SEDs and the spectroscopic data. Specifically, a 6 Myr-old population with $A_{V} \sim 3$ mag could reproduce well the spectroscopic data, but would not fit the photometric points.
}

The derived masses, ages, and extinctions for the two stellar populations in the ring sections are given in Table 8 together with the model fits for the spectroscopic data (which can be compared with the observations in Table 5) and bolometric luminosity. Fig. 6] shows the fits to the photometric SEDs. As can be seen from Table 8 the four ring sections have similar fitted properties. The young stellar population has a rather restricted age range $(\sim 5-6 \mathrm{Myr})$ and an average extinction of $A_{V} \sim 10 \mathrm{mag}$. The fitting of the age is basically driven by the EW of $\mathrm{Br} \gamma$ under the assumption that $A_{V}$ (stars) $=A_{V}$ (gas). However, if the extinction to the gas is higher than that to the stars (e.g., Calzetti et al. 2000), age would be an upper limit. The mass in young stars for each aperture is between $\sim 250$ and $500 \times 10^{6} \mathrm{M}_{\odot}$, comparable in most cases to the mass of the intermediate-age stellar population (see below). The only ring section with a mass in young stars significantly smaller than that of the intermediateage population is A4 (the eastern part of the ring), as suggested from the lack of bright MIR emission there (see $\S 6$ ) and the lower $\operatorname{Br} \gamma$ emission line flux (when compared to the other ring sections). The second stellar population in the ring sections has an intermediate age ( $\sim 17-35 \mathrm{Myr})$ and is less extincted $\left(A_{V} \sim 1.8 \mathrm{mag}\right)$. Interestingly, these properties are similar to those of the majority of the $1.1 \mu \mathrm{m}$-selected clusters (see $\S 6$ ).

We have also modelled the photometric SED and the spectroscopic properties of the whole ring, which corresponds to the ring area used by G95 (see Fig. 2 and Table 51). We used the $\operatorname{Br} \gamma$ flux given by G95 $\left(2.25 \times 10^{-14} \mathrm{erg} \mathrm{s}^{-1} \mathrm{~cm}^{-2}\right)$, and the EW of $\operatorname{Br} \gamma(9.9 \AA)$ as measured from the mean spectrum of the three (almost) non-overlapping ring sections (A1, A3 and A4) covered by UKIRT slits. Besides, we used the bolometric luminosity $\left(\mathrm{L}_{\mathrm{IR}} \sim \mathrm{L}_{\mathrm{bol}} \simeq 3 \times 10^{11} \mathrm{~L}_{\odot}\right.$, with a $50 \%$ of uncertainty) of the ring derived by G95 as an additional datapoint to fit. The results are presented in Table 8 and Fig. 7 We find ages of $5 \mathrm{Myr}$ and $14 \mathrm{Myr}$ for the young and intermediate-age populations, with $A_{V} \sim 11 \mathrm{mag}$ and $A_{V} \sim 2 \mathrm{mag}$, respectively. The fitted ages and extinctions are in agreement with those fitted for the ring sections, not surprisingly, as the three non-overlapping apertures, which have all similar fitted parameters, account for $\sim 80 \%$ of the fitted stellar mass of the ring (Table 9). Thus the ring sections covered by the UKIRT spectroscopy are representative of the ring as a whole. As can be seen from Table 8, the young stellar population accounts for about one-third of the stellar mass of the ring but, interestingly, is responsible for two-thirds of its bolometric luminosity.

We can independently estimate the extinction of the ionizing stellar population of the star-forming ring of NGC 7469. A number of authors (e.g., Calzetti et al. 2005; Alonso-Herrero et al. 2006a) have found a good correlation between the MIR and the extinctioncorrected $\mathrm{Pa} \alpha$ luminosities for star forming regions, starbursts, and LIRGs. Thus, using the $12 \mu \mathrm{m}$ vs. Pa $\alpha$ relation shown by Alonso-Herrero et al. (2006b) and the ring luminosity at $12.5 \mu \mathrm{m}$ ( 1 Jy) deduced from Soifer et al. (2003), we can estimate the number of ionizing photons (corrected for extinction) for the ring of $\mathrm{SF}$ and compare it with the observed $\mathrm{Br} \gamma$ flux. Assuming case B recombination (Hummer \& Storey 1987) and 
TABLE 8

Results of the $\chi^{2}$-Fitting of the SEDs and SPeCtroscopic Data of the Ring SECTIONS AND the whole Ring.

\begin{tabular}{|c|c|c|c|c|c|c|c|c|c|c|}
\hline \multirow[t]{2}{*}{ Reg. (a) } & \multicolumn{3}{|c|}{ Young pop. ${ }^{(b)}$} & \multicolumn{3}{|c|}{ Int-age pop. ${ }^{(c)}$} & \multirow[b]{2}{*}{$\begin{array}{c}\operatorname{Flux}_{\mathrm{Br} \gamma}{ }^{(\mathrm{g})} \\
\left(\operatorname{erg~s}^{-1} \mathrm{~cm}^{-2}\right)\end{array}$} & \multirow[b]{2}{*}{$\begin{array}{c}\mathrm{EW}_{\mathrm{Br} \gamma}{ }_{(\AA)}^{(\mathrm{h})} \\
(\AA)\end{array}$} & \multirow[b]{2}{*}{$\log \left(\mathrm{L}_{\mathrm{bol}} / \mathrm{L}_{\odot}\right)^{(\mathrm{i})}$} & \multirow[b]{2}{*}{$\chi^{2(j}$} \\
\hline & $\begin{array}{c}t^{(\mathrm{d})} \\
(\mathrm{Myr})\end{array}$ & $\begin{array}{l}A_{V}(\mathrm{e}) \\
(\mathrm{mag})\end{array}$ & $\begin{array}{c}m_{*}(\mathrm{f}) \\
\left(\times 10^{6} \mathrm{M}_{\odot}\right)\end{array}$ & $\begin{array}{c}t^{(\mathrm{d})} \\
(\mathrm{Myr})\end{array}$ & $\begin{array}{l}A_{V}(\mathrm{e}) \\
(\mathrm{mag})\end{array}$ & $\begin{array}{c}m_{*}(\mathrm{f}) \\
\left(\times 10^{6} \mathrm{M}_{\odot}\right)\end{array}$ & & & & \\
\hline A1 & $6.0 \pm_{0.5}^{0.5}$ & $9.50 \pm_{3.00}^{7.00}$ & $390 \pm_{100}^{340}$ & $17.0 \pm_{3.0}^{18.0}$ & $1.50 \pm_{0.50}^{0.25}$ & $310 \pm_{160}^{40}$ & $3.56 \times 10^{-15}$ & 10.5 & 11.03 & \\
\hline A2 & $5.5 \pm \pm_{0.5}^{0.5}$ & $\begin{array}{r}3.000 \\
13.00 \pm 7.00\end{array}$ & $\begin{array}{l}100 \\
340 \pm \\
3050\end{array}$ & $17.0 \pm 13.0$ & $2.50 \pm 0.250$ & $\begin{array}{l}450 \pm \\
40\end{array}$ & $3.83 \times 10^{-15}$ & 11.3 & 11.05 & \\
\hline A3 & $6.0 \pm_{0.5}^{0.5}$ & $11.50 \pm \frac{7.50}{6.50}$ & $480 \pm 5250$ & $35.0 \pm 20.0$ & $1.25 \pm_{0.25}^{0.50}$ & $\begin{array}{r}20 \\
430 \pm 200\end{array}$ & $3.69 \times 10^{-15}$ & 10.4 & 11.09 & \\
\hline $\mathrm{A} 4$ & $6.0 \pm 0.5$ & $7.50 \pm 12.50$ & $270 \pm 120$ & $17.0 \pm 13.0$ & $2.25 \pm_{0.50}^{0.25}$ & $\begin{array}{l}390 \pm 40 \\
50\end{array}$ & $3.03 \times 10^{-15}$ & 8.5 & 10.93 & 6. \\
\hline Ring & $5.0 \pm_{0.5}^{1: 8}$ & $10.50 \pm 7.50$ & $860 \pm_{380}^{1230}$ & $14.0 \pm_{1.0}^{1.0}$ & $1.75 \pm_{0.25}^{0: 25}$ & $1830 \pm 70$ & $2.32 \times 10^{-14}$ & 9.6 & 11.58 & 9.7 \\
\hline
\end{tabular}

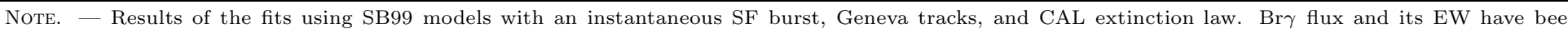

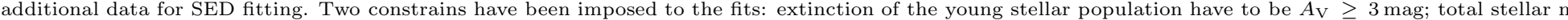

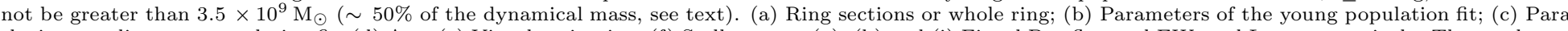

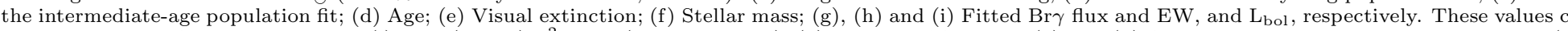

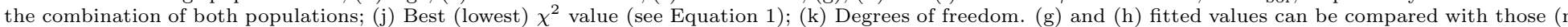
introduced for the fit (see Table 6).

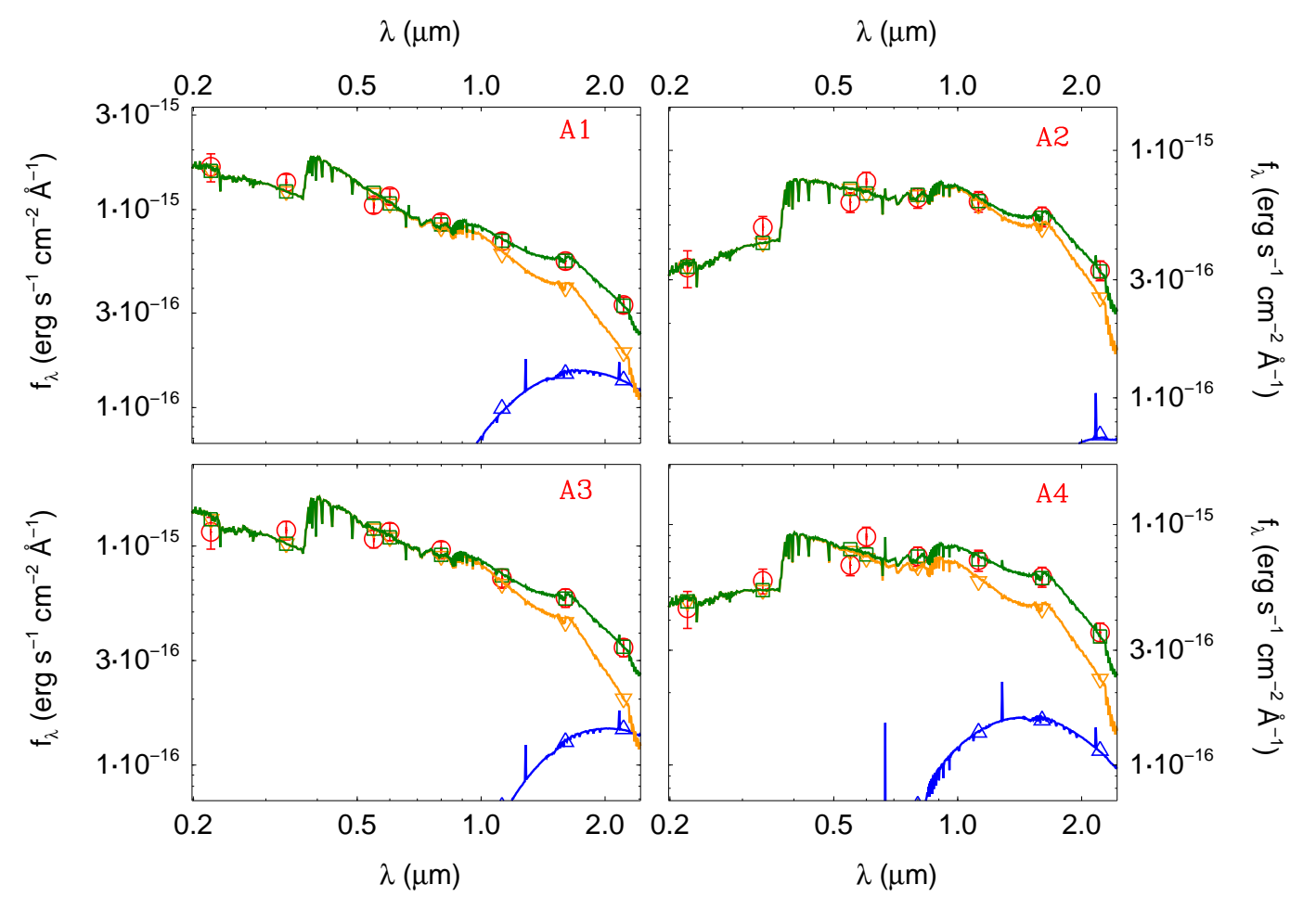

FIG. 6. - Fits to the SEDs (and spectroscopic data, not shown) of the four ring sections (see locations in Fig. 22). We imposed the extinction of the young stellar population to be $A_{\mathrm{V}} \geq 3 \mathrm{mag}$. The observational datapoints are shown as (red) open circles with their corresponding $1 \sigma$ uncertainties. The SED of the young stellar population is shown in blue, the intermediate-age population in orange, and the total (sum) fitted SED in green. The (blue) triangles, inverted (orange) triangles, and (green) squares are the model photometric points (SB99 SEDs convolved with HST-filter throughputs) of the best fit for each population, respectively. [See the electronic edition of the Journal for a color version of this figure.]

using the CAL extinction law we derive $A_{V} \sim 13 \mathrm{mag}$ $\left(A_{\mathrm{Br} \gamma} \sim 1.25 \mathrm{mag}\right)$. This high value of the extinction confirms independently (and agrees with) the existence of a deeply obscured young population inferred from the SED analysis of the ring sections and the whole ring (\$5.1).

\subsection{Additional Spectroscopic Indicators}

The He I $2.06 \mu \mathrm{m} / \mathrm{Br} \gamma$ line ratio has been used as an indicator of the effective temperature of the ionizing stellar population as well as a means of detecting the presence of massive stars and constraining the upper mass cutoff of the IMF. Under the assumptions made in this work for the IMF and metallicity, we can use this line ratio as an indicator of the age of the SF processes. For the observed range of the He I $2.06 \mu \mathrm{m} / \mathrm{Br} \gamma$ line ratio $(0.22-0.29$ for the spectroscopic apertures, see Table 6, and 0.29 for the ring, see G95) the age of the young population is approximately $\simeq 5.5-6 \mathrm{Myr}$ (using Fig. 3 of Rigby \& Rieke 2004 


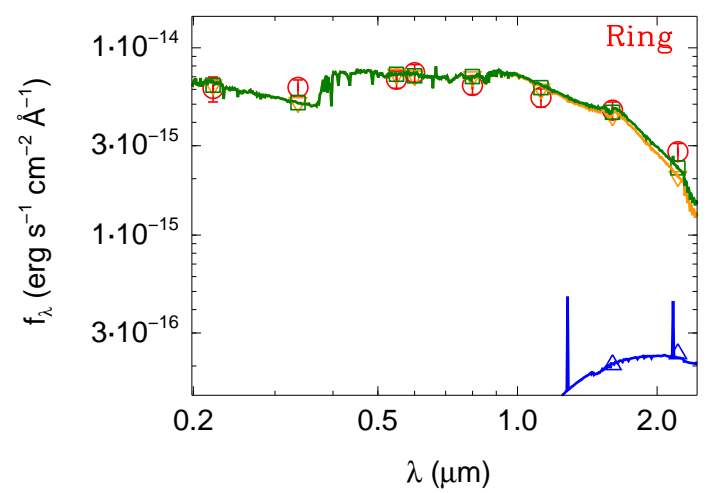

FIG. 7.- Fit to the whole ring SED (and spectroscopic data, not shown). We have used the bolometric luminosity of the ring (G95) as an additional datapoint (see $\S 5.1$ for details). The rest (constraints, symbols, and colors) are the same as in Fig 6] [See the electronic edition of the Journal for a color version of this figure.]

for a Salpeter IMF, solar metallicity, instantaneous burst and $M_{\text {up }}=100 \mathrm{M}_{\odot}$ ), in agreement with our previous findings (see $\S 5.1$ ). However, we take these results with caution as Shields (1993) and Lumsden et al. (2001) have demonstrated that this ratio has a complex dependence with the effective temperature and other $\mathrm{H}$ II region parameters, and cannot be used as an accurate indicator of the effective temperature (Rigby \& Rieke 2004).

Using SB99 models, the observed photometric CO index (see Table 6) is consistent with a stellar population with an age of $8-9 \mathrm{Myr}$ or $50-100 \mathrm{Myr}$. Our results would be in agreement with the former interval if we consider that the two stellar populations fitted in $\S 5.1$ ( $\sim 6$ Myr and $\sim 17-35$ Myr $)$ would generate an intermediate $\mathrm{CO}$ index, representative of the mixed populations. However, as discussed by Origlia \& Oliva (2000) the greatest uncertainty in deriving ages from $\mathrm{CO}$ indices is the stellar evolutionary tracks. This problem is alleviated if the models use observational NIR stellar libraries (see e.g., Förster-Schreiber et al. 2000).

Summarizing, within the uncertainties of the models, the ages suggested by the other NIR spectroscopic indicators of the ring sections agree well with the ages derived from the photometric $\mathrm{SED}+\mathrm{Br} \gamma$ fits discussed in the previous section, and lead to a consistent result which we will now place in a wider context.

\section{DISCUSSION}

\subsection{Contribution of the $1.1 \mu \mathrm{m}$-selected Star Clusters to} the Integrated Emission

As we saw in $\S 4$ and 5.1 most of the $1.1 \mu$ m-selected star clusters have properties similar to the integrated intermediate-age stellar population inferred for the ring. However, the flux contribution of the clusters to the ring sections and the whole ring decreases with wavelength (Table 91). For instance, the cluster flux contribution to the A1 aperture is $70 \%$ (40\% for the other ring sections) in the UV and only $\sim 10 \%$ in the NIR. This could be in part a spatial resolution effect. Since the spatial resolution of the UV image is lower than that of the $1.1 \mu \mathrm{m}$ image, it is possible that an unresolved UV cluster could actually be an aggregation of clusters when observed at higher spatial resolution. This is the case for some UV and NIR clusters which have multiple cross-identifications in the higher resolution F330W and F606W images (see $\S 2.1 .1$ ). However, the UV and the $2.2 \mu \mathrm{m}$ images have similar spatial resolutions, but the flux contribution of the clusters varies significantly between these two wavelengths. This suggests that differences in spatial resolution are not the main cause. It is more likely that the decreasing flux contribution (from UV to NIR) of the star clusters to the ring sections could be due to the existence of an evolved stellar population not spatially resolved as individual clusters, which would make up a significant contribution to the NIR emission, but almost none to the UV continuum emission.

In terms of the stellar masses, the $1.1 \mu \mathrm{m}$-selected clusters only represent a minimal contribution to the total stellar mass $(10 \%$ of the intermediate-age stellar population) within the ring sections. However, the images with the highest spatial resolutions (ACS F330W and WFPC2 F606W) reveal a larger number of clusters than those detected at $1.1 \mu \mathrm{m}$ (Fig. 1 and Table 3) whose properties are presumably similar to those described in $\S 4$. Given the similar ages and extinctions of the integrated intermediate-age stellar population detected in the ring and most of the $1.1 \mu \mathrm{m}$ star clusters, it is likely that this ring population is also made up of less massive clusters $\left(<1 \times 10^{6} \mathrm{M}_{\odot}\right)$, not resolved in the NICMOS F110W image. As such, the majority of the $1.1 \mu \mathrm{m}$-selected clusters would be the most massive (and thus easier to detect) examples of this intermediate-age stellar population.

\subsection{Gas/dust Distribution and its Relation with the Stellar Populations}

The existence of large amounts of obscuring gas and dust in LIRGs (and ULIRGs), and the major role played by the complexity of their spatial structure have been known for a long time (e.g., Sanders et al. 1986; Scoville et al. 2000; Bushouse et al. 2002; Alonso-Herrero et al. 2006a, among many others). This is indeed the case for NGC 7469. The large-scale HST imaging (Malkan, Gorjian \& Tam 1998; Scoville et al. 2000) of NGC 7469 shows that the ring has a dusty clumpy morphology embedded in a spiral-like structure with prominent dust lanes (Fig. 8 and Scoville et al. 2000; Martini et al. 2003). A large-scale (several kpc) stellar bar has been detected by Knapen et al. (2000). In addition, the CO 2-1 map of Davies et al. (2004) reveals the presence of molecular gas in the rims of the ring of SF where the dust lanes are and, more interestingly, forming a nuclear gas bar inside the ring, crossing the nucleus (see their Fig. 3).

In moderately dusty galaxies, and specially in LIRGs, the spatial locations of the young stellar populations can be inferred from their MIR emission (see, among others, Soifer et al. 2001; Helou et al. 2004; Calzetti et al. 2005; Alonso-Herrero et al. 2006b). The $12.5 \mu \mathrm{m}$ map of NGC 7469 from Soifer et al. (2003, see also the Br $\gamma$ map of G95) shows bright MIR peaks in the northeast, south and southwest regions of the ring (see Fig. 9). In fact, the ends of the nuclear molecualr gas bar coincide with the position of these bright MIR peaks (obscured regions of ongoing SF), and these probably with the location of 
TABLE 9

CONTRIBUtion OF THE $1.1 \mu \mathrm{M}$-SELECTED ClUSTERS TO THE RING SECTIONS AND WhOlE RING.

\begin{tabular}{|c|c|c|c|c|c|c|c|c|c|c|}
\hline Reg. (a) & Clusters $^{(\mathrm{b})}$ & $\mathrm{F} 218 \mathrm{~W}^{(\mathrm{c})}$ & F330W ${ }^{(c)}$ & $\mathrm{F} 547 \mathrm{M}^{(\mathrm{c})}$ & $\mathrm{F} 606 \mathrm{~W}^{(\mathrm{c})}$ & $\mathrm{F} 814 \mathrm{~W}^{(\mathrm{c})}$ & $\mathrm{F} 110 \mathrm{~W}^{(\mathrm{c})}$ & $\mathrm{F} 160 \mathrm{~W}^{(\mathrm{c})}$ & $\mathrm{F} 222 \mathrm{M}^{(\mathrm{c})}$ & Mass contrib. $(\mathrm{d})$ \\
\hline $\mathrm{A} 1$ & $\begin{array}{c}\mathrm{C} 1_{1 \#}, \mathrm{C} 4_{1 \#}, \mathrm{C} 7_{1 \#}, \\
\mathrm{C} 13, \mathrm{C} 15\end{array}$ & $70 \%$ & $36 \%$ & $18 \%$ & $20 \%$ & $13 \%$ & $12 \%$ & $10 \%$ & $13 \%$ & $5 \%$ \\
\hline A2 & $\mathrm{C} 3, \mathrm{C} 6_{1 \#}, \mathrm{C} 8_{1 \#}$ & $42 \%$ & & $10 \%$ & 1607 & $6 \%$ & & & & \\
\hline A3 & $\mathrm{C} 2, \mathrm{C} 5, \mathrm{C} 8_{1 \#}$ & $42 \%$ & $27 \%$ & $10 \%$ & $16 \%$ & $6 \%$ & $11 \%$ & $11 \%$ & $12 \%$ & $5 \%$ \\
\hline A4 & $\begin{array}{l}\mathrm{C} 24_{1 \#}, \mathrm{C} 25_{1 \#} \\
\mathrm{C} 3, \mathrm{C} 6_{1 \#}, \mathrm{C} 11,\end{array}$ & $44 \%$ & $33 \%$ & $18 \%$ & $20 \%$ & $9 \%$ & $14 \%$ & $12 \%$ & $12 \%$ & $6 \%$ \\
\hline & $\mathrm{C} 22, \mathrm{C} 26, \mathrm{C} 27$ & $45 \%$ & $25 \%$ & $14 \%$ & $13 \%$ & $7 \%$ & $12 \%$ & $9 \%$ & $11 \%$ & $5 \%$ \\
\hline Ring & $\mathrm{A} 1, \mathrm{~A} 3, \mathrm{~A} 4$ & $53 \%$ & $51 \%$ & $41 \%$ & $43 \%$ & $40 \%$ & $38 \%$ & $37 \%$ & $36 \%$ & $80 \%$ \\
\hline
\end{tabular}

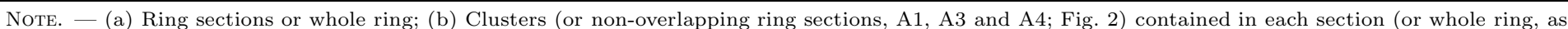

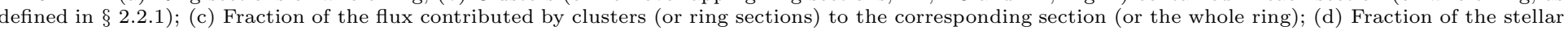

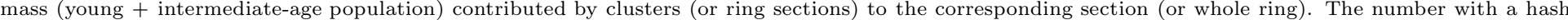

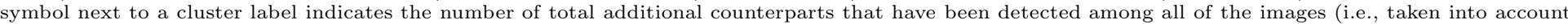
all wavelengths; see Table 2 for details).

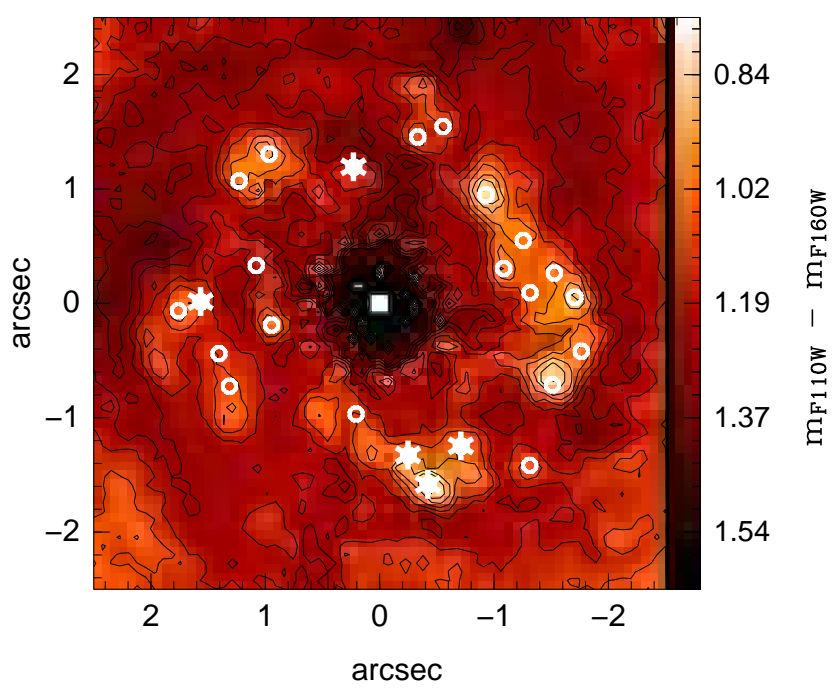

FIG. 8.- $m_{\mathrm{F} 110 \mathrm{~W}}-m_{\mathrm{F} 160 \mathrm{~W}}$ color map (equivalent to a $J-H$ color map). Colors redder (darker) than $m_{\mathrm{F} 110 \mathrm{~W}}-m_{\mathrm{F} 160 \mathrm{~W}} \sim 1.03$ (typical of an old stellar population, although younger ionizing stellar populations will be bluer, see Alonso-Herrero et al. 2006a) are indicative of highly extincted regions. The young and intermediateage $1.1 \mu \mathrm{m}$-selected star clusters are marked with star symbols and open circles, respectively. Because of the central region $\left(\mathrm{r} \lesssim 0^{\prime \prime} .5\right)$ is strongly affected by the subtraction of the nucleus, it does not show any real extinction variation. [See the electronic edition of the Journal for a color version of this figure.]

the Inner Lindblad Resonance(s) of the large-scale stellar bar (Knapen et al. 1995, Jogee, Scoville \& Kenney 2005). The sketch shown in Fig. 10 summarizes the complexity of the spatial distribution of the stars, dust, and gas in the star-forming ring of NGC 7469.

As can been seen from Fig. 9, there is a clear spatial anti-correlation between the positions of the UV-opticalNIR clusters and the bright MIR emitting regions. The radio (Colina et al. 2001) and the MIR peaks in the ring of NGC 7469, on the other hand, are spatially coincindent, as found in other LIRGs (Soifer et al. 2001). These displacements between the UV-optical-NIR con- tinuum peaks and MIR/radio bright emitting regions are real and correspond to distances of approximately $160 \mathrm{pc}$ $(0,5)$.

This lack of spatial coincidence between UV-opticalNIR and MIR continua is in agreement with our model of two stellar populations to fit the integrated properties of the ring (and ring sections). The UV-optical-NIR continuum (cluster and unresolved emission) traces mostly the mildly extincted intermediate-age $(\lesssim 100 \mathrm{Myr})$ population, whereas the MIR and radio peaks reveal the regions where the most obscured and youngest $(\lesssim 8-10 \mathrm{Myr})$ $\mathrm{SF}$ is taking place. Interestingly, this agrees with the fact that the $1.1 \mu \mathrm{m}$-selected clusters, which mostly trace (and are representative of) the intermediate-age population of the ring $(\S 6.1)$, are indeed located in low extinction regions (color map, Fig. 8) that in general do not coincide with the bright MIR peaks nor with the molecular gas and dust lanes in the ring (see Figs. 9] and [10). That is, most of the clusters have been detected where no current but rather recent ( $\gtrsim 8-10 \mathrm{Myr}$ ) SF and low extinction are expected, which agrees with the parameters found for them ( 14 Myr and $1.5 \mathrm{mag}$ of visual extinction). The only exception are the few young $1.1 \mu \mathrm{m}$ star clusters that are located in the southern part of the ring (where molecular gas, MIR emisssion, and NIR continuum overlap), which could be the most massive (luminous), less-extincted examples of the integrated young stellar population, as already inferred from the SED fitting of the ring sections $(\S 4)$. However, due to the small number of young $1.1 \mu \mathrm{m}$-selected clusters, they might not be statistically representative of the whole young population.

We conclude that in the ring of NGC 7469, the UVoptical-NIR broad-band continuum emission reflects the recent $\mathrm{SF}(\lesssim 100 \mathrm{Myr}$ ), but not necessarily the ongoing $(\lesssim 8-10 \mathrm{Myr})$ obscured SF. The location and properties of the youngest population are only revealed by the MIR and radio emission, as well as the NIR Br $\gamma$ hydrogen recombination line. In turn, because of the spatially variable and strong obscuration in the ring of this galaxy, the $1.1 \mu \mathrm{m}$-selected star clusters mostly trace the intermediate-age, low-extincted stellar population.

\section{CONCLUSIONS}



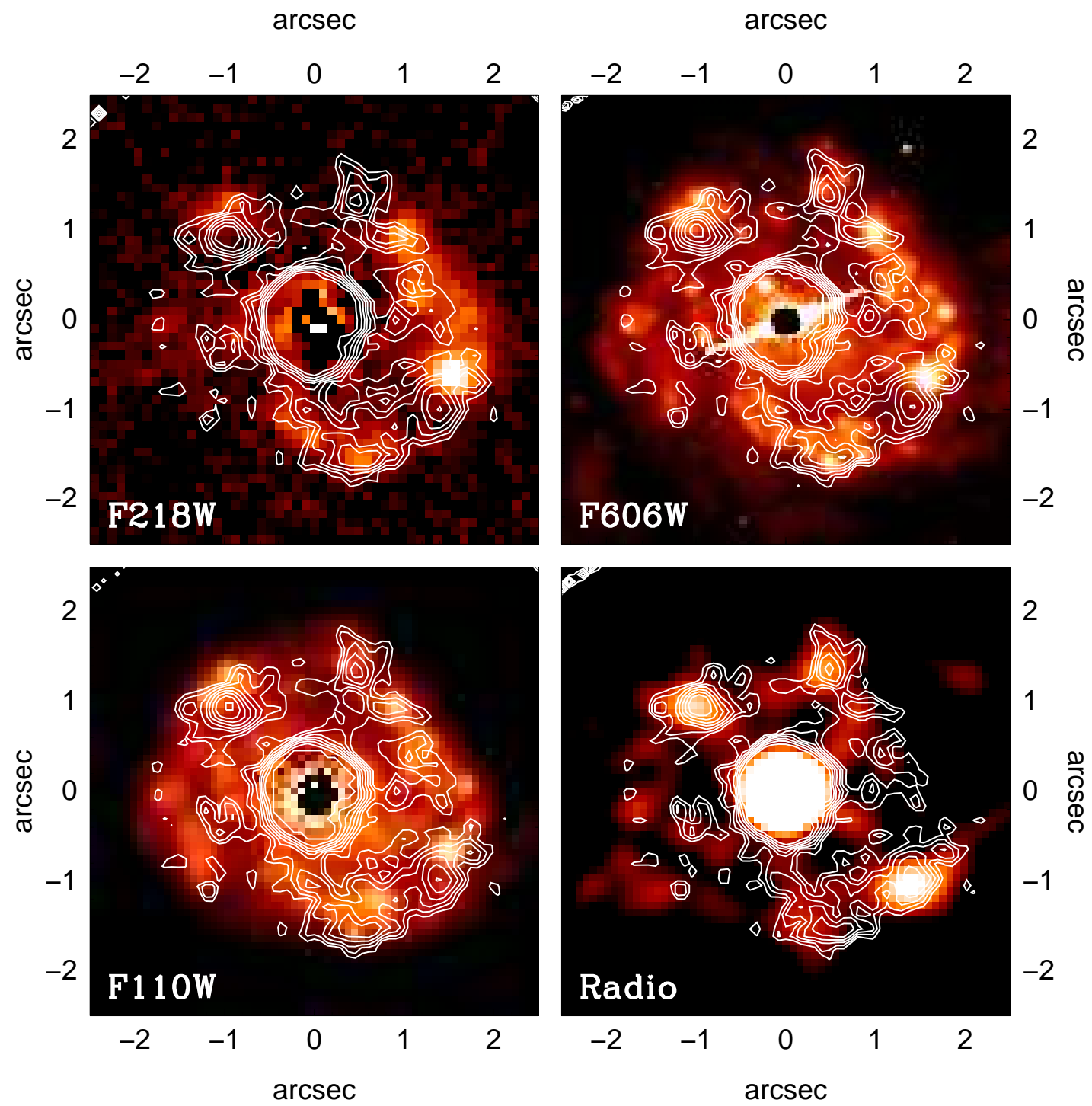

FIG. 9.- In all the images the contours are the MIR $12.5 \mu \mathrm{m}$ emission (Soifer et al. 2003) superimposed on the UV F218W (left, top panel), optical F606W (right, top panel), NIR $1.1 \mu \mathrm{m}$ (left, bottom panel), and radio $8.4 \mathrm{GHz}$ (right, bottom panel, Colina et al. 2001; obtained in Nov 1999) images. Note the excellent spatial correspondence between the MIR and radio emissions whereas, in general, the locations of brightest NIR, optical, and UV clusters (except those to the south of the nucleus, see $\S 6.2$ ) do not correspond with the brightest peaks of MIR emission. [See the electronic edition of the Journal for a color version of this figure.]

We have presented a multi-wavelength HST UVthrough-NIR imaging analysis of the circumnuclear ring of SF of NGC 7469, together with new ground-based NIR spectroscopy at four locations along the ring. These high spatial resolution data have allowed us to study the SF properties at different spatial scales, ranging from individual star clusters (tens of parsecs) to ring sections (hundreds of parsecs) and the ring as a whole (diameter of $\simeq 1.6 \mathrm{kpc}$ ).

At the smallest scales we have selected 30 star clusters at $1.1 \mu \mathrm{m}$. The fitting of their SEDs reveals the presence of two stellar populations. Most (75\%) of the clusters are of intermediate age $(8-20 \mathrm{Myr})$ and have low extinction $\left(A_{V} \sim 1.25 \mathrm{mag}\right)$. The others are younger $(\sim 1-3 \mathrm{Myr})$ and are moderately extincted $\left(A_{V} \sim 3 \mathrm{mag}\right)$. Most clusters have stellar masses within the range $1-10 \times 10^{6} \mathrm{M}_{\odot}$, although more massive clusters or aggregates of clusters $\left(10-15 \times 10^{6} \mathrm{M}_{\odot}\right)$ are also found.

On the kiloparsec scale (ring sections and whole ring), the photometric SEDs and spectroscopic indicators (in particular $\operatorname{Br} \gamma$ luminosity and EW) were fitted with two distinct stellar populations: a young $(\sim 5-6 \mathrm{Myr})$, highly obscured $\left(A_{V} \simeq 7-13 \mathrm{mag}\right)$ population, and an intermediate-age $(\sim 14-35 \mathrm{Myr})$, less extincted $\left(A_{V} \sim\right.$ $1.8 \mathrm{mag}$ ) population. A single stellar population could not account for all the observed properties. The young ionizing population would be responsible for the bright $\mathrm{Br} \gamma$ line emission and the MIR and radio continuum emission peaks. This young population is generally not coincident with the UV-optical-NIR continuum emitting regions and the majority of the intermediate-age clusters. Moreover, the brightest MIR/radio regions coincide 


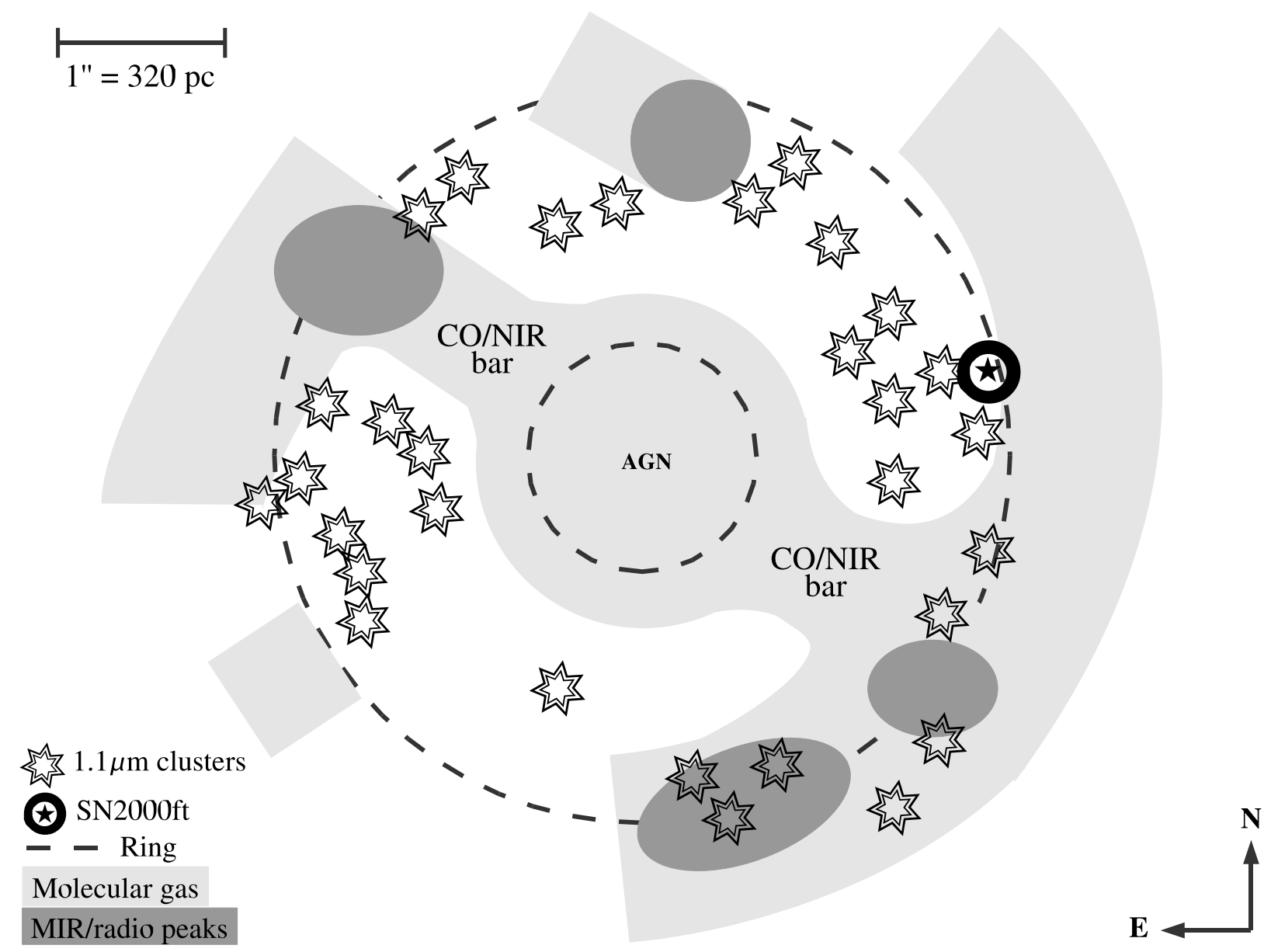

FIG. 10.- Sketch of the nuclear region of NGC 7469. The approximate locations of the $1.1 \mu$ m-selected star clusters are marked with stars. The places where there is molecular gas (traced by CO J=2-1 emission) are shaded in light gray, whereas the most intense MIR/radio emitting regions are shaded in dark gray. The location of SN2000ft (Colina et al. 2001, 2007) is shown as a black star.

with the ends of the nuclear molecuar gas bar detected in CO. This young population of ionizing stars, although it only represents one-third of the stellar mass of the ring, accounts for a large fraction (about $2 / 3$ ) of its IR luminosity. The $1.1 \mu \mathrm{m}$-selected clusters, although making up a small fraction of the ring stellar mass, are representative of the integrated intermediate-age stellar population responsible for the UV-optical-NIR continuum emission. On the other hand, the younger $(\sim 1-3 \mathrm{Myr})$ clusters could be the less extincted, massive examples of the young ionizing population traced by the MIR continuum and $\operatorname{Br} \gamma$ emission line.

In conclusion, multi-wavelength data (UV-throughradio imaging and NIR spectroscopy) have allowed us to characterize the SF properties of both the star clusters and the ring of SF of NGC 7469. In the case of this LIRG, and other dusty galaxies, these types of observations are critically needed to discern and study the current (traced by MIR/radio continuum emission and NIR/MIR emission lines) and the recent (detected through UV-opticalNIR continuum) SF taking place in the dusty environments commonly found in LIRGs and ULIRGs.
We thank B. T. Soifer and E. Egami for providing us with the MIR image of NGC 7469. This work has been supported by the Plan Nacional del Espacio under grant ESP2005-01480. TDS acknowledges support from the Consejo Superior de Investigaciones Científicas under grant I3P-BPD-2004. JHK acknowledges support from the Leverhulme Trust in the form of a Leverhulme Research Fellowship. AAH thanks the Centre for Astrophysics Research at the University of Hertfordshire for hospitality. We have made use of observations made with the NASA/ESA Hubble Space Telescope, obtained from the data archive at the Space Telescope Science Institute (STScI). The STScI is operated by the Association of Universities for Research in Astronomy, Inc. under NASA contract NAS 5-26555. This research has made use of the NASA/IPAC Extragalactic Database (NED), which is operated by the Jet Propulsion Laboratory, California Institute of Technology, under contract with the National Aeronautics and Space Administration, and of NASA's Astrophysics Data System (ADS) abstract service. The United Kingdom Infrared Telescope is operated by the Joint Astronomy Centre on behalf of the U.K. Particle Physics and Astronomy Research Council. 


\section{REFERENCES}

Alberdi, A., Colina, L., Torrelles, J. M., Panagia, N., Wilson, A. S., Garrington, S. T. 2006, ApJ, 638, 938

Allard, E. L., Knapen, J. H., Peletier, R. F., \& Sarzi, M. 2006, MNRAS, 371, 1087

Allard, E. L., Sarzi, M., Knapen, J. H., \& Mazzuca, L. M. 2007, MNRAS, submitted

Alonso-Herrero, A., Rieke, G. H., Rieke, M. J., \& Scoville, N. Z 2000, ApJ, 532, 845

Alonso-Herrero, A., Ryder, S. D., \& Knapen, J. H. 2001a, MNRAS, 322,757

Alonso-Herrero, A., Engelbracht, C. W., Rieke, M. J., Rieke, G. H., \& Quillen, A. C. 2001b, ApJ, 546, 952

Alonso-Herrero, A., Rieke, G. H., Rieke M. J., \& Scoville, N. Z. 2002, AJ, 124, 166

Alonso-Herrero, A., Rieke, G. H., Rieke, M. J., Colina, L., PérezGonzález, P. G., \& Ryder, S. D. 2006a, ApJ, 650, 835

Alonso-Herrero, A., Colina, L., Packham, C., Díaz-Santos, T., Rieke, G. H., Radomski, J. T., \& Telesco, C. 2006b, ApJL, 652, 83

Bertin, E., \& Arnouts, S. 1996, A\&AS, 117, 393

Bastian, N., Gieles, M., Lamers, H. J. G. L. M., Scheepmaker, R A., \& de Grijs, R. 2005, A\&A, 431, 905

Bevington, P. R., \& Robinson, D. K. 2003. Data reduction and error analysis for the physical sciences (3rd ed.; New York: McGrawHill)

Bik A., Lamers H. J. G. L. M., Bastian N., Panagia N., \& Romaniello M. 2003, A\&A, 397, 473

Burbidge, E. M., Burbidge, G. R., \& Prendergast, K. H. 1963, ApJ, 137, 1022

Bushouse, H. A., et al. 2002, ApJS, 138, 1

Buta, R., Treuthardt, P. M., Byrd, G. G., \& Crocker, D. A. 2000, AJ, 120, 1289

Calzetti, D. 1997, AJ, 113, $162(\mathrm{CAL})$

Calzetti, D. et al. 2005, ApJ, 633, 871

Colina, L., Alberdi, A., Torrelles, J. M., Panagia, N., \& Wilson A. S. 2001, ApJ, 553, 19

Colina, L., Díaz-Santos, T., Alonso-Herrero, A., Alberdi, A. Torrelles, J. M., Panagia, N., \& Wilson A. S. 2007, ApJL, submitted

Cutri, R. M., Rieke, G. H., Tokunaga, A. T., Willner, S. P., \& Rudy, R. J. 1984, ApJ, 280, 521

Dahari, O. 1985, AJ, 90, 1772

Davies, R. I., Tacconi, L. J., \& Genzel, R. 2004, ApJ, 602, 148

de Grijs, R., Anders, P., Lamers, H. J. G. L. M., Bastian, N. Fritze-v. Alvensleben, U., Parmentier, G., Sharina, M. E., \& Yi, S. 2005, MNRAS, 359,874

de Grijs, R., Parmentier, G., \& Lamers, H. J. G. L. M. 2005 MNRAS, 364, 1054

Downes, D., \& Solomon, P. M. 1998, ApJ, 507, 615

Doyon, R., Joseph, R. D., \& Wright, G. S. 1994, ApJ, 421, 101

Förster-Schreiber, N. M. 2000, NewAR, 44, 263

Frogel, J. A., Persson, S. E., Aaronson, M., \& Matthews, K. 1978, ApJ, 220, 75

Gao, Y., \& Solomon, P. M. 2004, ApJ, 606, 271

García-Marín, M., Colina, L., Arribas, S., Alonso-Herrero, A., \& Mediavilla, E. 2006, ApJ, 650, 850

Genzel R., Weitzel L., Tacconi-Garman L. E., Blietz M., Cameron M., Krabbe A., Lutz D., Sternberg A., 1995, ApJ, 444, 129 (G95)

Gorjian, V., Werner, M. W., Jarrett, T. H., Cole, D. M., \& Ressler, M. E. 2004, ApJ, 605,156

Heckman, T. M., Beckwith, S., Blitz, L., Skrutskie, M., \& Wilson, A. S. 1986, ApJ, 305, 157

Helou, G. et al. 2004, ApJS, 154, 253

Hummer, D. G., \& Storey, P. J. 1987, MNRAS, 224, 801

Jogee, S., Scoville, N., \& Kenney, J. D. P. 2005, ApJ, 630, 837

Kennicutt, R. C. Jr. 1998, ARA\&A, 36, 189

Knapen, J. H., Beckman, J. E., Heller, C. H., Shlosman, I., \& de Jong, R. S. 1995, ApJ, 454, 623

Knapen, J. H., Shlosman, I., \& Peletier, R. F. 2000, ApJ, 529, 93

Knapen, J. H. 2004, Astrophy. Sp. Sc. Library, 319, 189
Krist, J., Golimowski, D. A., Schroeder, D. J., \& Henry, T. J. 1998, PASP, 110, 1046

Laine, S., Shlosman, I., Knapen, J. H., \& Peletier, R. F. 2002, ApJ, 567,97

Leitherer, C., Schaerer, D., Goldader, J. D., Delgado, R. M. G., Robert, C., Kune, D. F., de Mello, D. F., Devost, D., Heckman, T. M. 1999, ApJ, 123, 3, SB99

Lonsdale, C. J., Farrah, D., \& Smith, H. E. 2006, in press, to published in "Astrophysics Update 2 - topical and timely reviews on astronomy and astrophysics". Ed. John W. Mason. Springer/Praxis books (astro-ph/0603031)

Lumsden, S. L., Puxley, P. J., \& Hoare, M. G. 2001, MNRAS, 320, 83

Malkan, M. A., Gorjian, V., \& Tam, R. 1998, ApJS, 117, 25

Maoz, D., Barth, A. J., Sternberg, A., Filippenko, A. V., Ho, L. C., Macchetto, F. D., Rix, H.-W., \& Schneider, D. P. 1996, AJ, 111,2248

Maoz, D., Barth, A. J., Ho, L. C., Sternberg, A., \& Filippenko, A. V. 2001, AJ, 121, 3048

Martini, P., Regan, M. W., Mulchaey, J. S., \& Pogge, R. W. 2003, ApJS, 146, 353

Mauder, W., Weigelt, G., Appenzeller, I., \& Wagner, S. J. 1994, A\&A, 285, 44

Mazzuca, L. M., Sarzi, M., Knapen, J. H., Veilleux, S., \& Swaters, R. 2006, ApJL, 649, 79

Meixner, M., Puchalsky, R., Blitz, L., Wright, M., \& Heckman, T. 1990, ApJ, 354, 158

Miles, J. W., Houck, J. R., \& Hayward, T. L. 1994, ApJ, 425, 37

Mountain, C. M., Robertson, D. J., Lee, T. J., \& Wade, R. 1990. Instrumentation in astronomy VII; Proceedings of the Meeting, Tucson, AZ, Feb. 13-17, 1990 (A91-29601 11-35). Bellingham, WA, Society of Photo-Optical Instrumentation Engineers. Pag. 25-33.

Murphy, T. W., Jr., Soifer, B. T., Matthews, K., Armus, L. 2001, ApJ, 559, 201

Origlia, L., \& Oliva, E. 2000, A\&A, 357, 61

Papadopoulos, P. P., \& Allen, M. L. 2000, ApJ, 537, 631

Rigby, J. R., \& Rieke, G. H. 2004, ApJ, 606, 237

Ryder, S. D., Knapen J. H., \& Takamiya M. 2001, MNRAS, 323 663

Salpeter, E. E. 1955, ApJ, 121, 161

Sanders, D. B. et al. 1986, ApJ, 305, L45

Sanders, D. B., \& Mirabel, I. F. 1996, ARA\&A, 34, 749

Scoville, N. Z., Sargent, A. I., Sanders, D. B., \& Soifer, B. T. 1991 , ApJ, 366, 5

Scoville, N. Z., Evans, A. S., Thompson, R., Rieke, M., Hines, D. C., Low, F. J.,Dinshaw, N., Surace, J. A., \& Armus, L. 2000, AJ, 119,991

Shields, J. C. 1993, ApJ, 419, 181

Soifer, B. T., Neugebauer, G. Matthews, K., Egami, E., Weinberger, A. J., Ressler, M., Scoville, N. Z., Stolovy, S. R., Condon, J. J., \& Becklin, E. E. 2001, AJ, 122, 1213

Soifer, B. T., Bock, J. J., Marsh, K., Neugebauer, G., Matthews, K., Egami, E., \& Armus L. 2003, AJ, 126, 143

Surace, J. A., Sanders, D. B., Evans, A. S. 2000, ApJ, 529, 170

Stetson, P. B. 1987, PASP, 99, 191

Thilker, D. A., Braun, R., \& Walterbos, R. A. 2000, AJ, 120, 3070

Ulvestad, J. S., Wilson, A. S., \& Sramek, R. A. 1981, ApJ, 247, 419

Vázquez, G. A., \& Leitherer, C. 2005, ApJ, 621, 695

Wilson, A. S., Daldwin, J. A., Sun, S-D., \& Wright, A. E. 1986, ApJ, 310, 121

Wilson, A. S., Helfer, T. T., Haniff, C. A., \& Ward, M. J. 1991, ApJ, 381, 79

Wilson, C. D., Harris, W. E., Longden, R., \& Scoville, N. Z. 2006, ApJ, 641, 763

Zhang, Q., \& Fall, S. M. 1999, ApJ, 527, 81 